\title{
INTELIGENCIA ARTIFICIAL
}

http://journal.iberamia.org/

\section{Completeness by Modal Definitions. Application to the Epistemic Logic With Hypotheses}

\author{
Levan Uridia and Dirk Walther \\ TSU Razmadze Mathematical Institute, Tbilisi, Georgia \\ l.uridia@freeuni.edu.ge \\ DNV GL, Norway \\ dirk.walther@dnvgl.com
}

\begin{abstract}
We investigate the variant of epistemic logic S5 for reasoning about knowledge under hypotheses. The logic is equipped with a modal operator of necessity that can be parameterized with a hypothesis representing background assumptions. The modal operator can be described as relative necessity and the resulting logic turns out to be a variant of Chellas' Conditional Logic. We present an axiomatization of the logic and its extension with the common knowledge operator and distributed knowledge operator. We show that the logics are decidable, complete w.r.t. Kripke as well as topological structures. The topological completeness results are obtained by utilizing the Alexandroff connection between preorders and Alexandroff spaces.
\end{abstract}

Keywords: Modal epistemic logic, Kripke completeness, Topological completeness, Common/Distributive knowledge operators

\section{Introduction}

This paper is a combination and extension of the results from [24, 25] and [26]. We apply techniques from 24] for obtaining Kripke completeness results for certain extensions of a modal logics, and a technique for obtaining topological completeness from Kripke completeness to the extensions of modal logic of Hypothesis. Consider extensions of a modal logic $L$ with modal definitions of the form

$$
\boxplus p \leftrightarrow \varphi(p),
$$

where ' $\boxplus$ ' is a fresh box-modality, and $p$ is a proposition occurring in $\varphi$. That is, the modality $\boxplus$ is defined in terms of $\varphi$ in which $\boxplus$ does not occur. In the paper 24] authors point out the conditions on $\varphi$ under which we obtain Kripke completeness of the extended logic. It is stated as an interesting open problem to find a syntactic characterisation of modal definitions that give rise to a relational semantics. The related problem of characterising elementary formulas (i.e., modal formulas that define a first-order frame property) has been studied extensively; see, e.g., 21, [13, 27. However, elementarity is neither a necessary nor sufficient criterion for a modal formula to be used in relational modal definitions.

The idea to add modal definitions to existing normal modal logics is quite common, e.g., for (dynamic) epistemic logics. The following formulas are examples of modal definitions: $E_{A} p \leftrightarrow \bigwedge_{a \in A} \square_{a} p$ is the axiom for 'everyone knows' in epistemic logic, i.e., every agent in the group $A$ knows $p$ [11]; [! $\varphi] p \leftrightarrow(\varphi \rightarrow p)$ is the reduction axiom for the announcement operator [! $\varphi]$ in Public Announcement Logic [19], 28]; $\square_{S 4} p \leftrightarrow \square_{\mathrm{K} 4} p \wedge p$ is a definition of an S4-box modality in terms of an K4-box modality [9]; and [ $\left.\varphi\right] p \leftrightarrow$ $\square p \vee(\varphi \wedge \square(\varphi \rightarrow p))$ is the reduction axiom used for the logic $\mathrm{S}^{r}$ for reasoning under hypotheses in [23]. 
It is shown that one can obtain a finite axiomatisation of normal modal logics extended with relational modal definitions in a straightforward way. We illustrate this technique with two extensions of the modal logic S5. We consider the epistemic logic for reasoning about knowledge under hypotheses from [23]. The resulting logic $\mathrm{S}^{r}$ is an extension of the epistemic logic S5 with a modal operator '[.]' that can be parameterized with a hypothesis. The operator can be described as relative necessity, a notion already used by Chellas to describe conditionality [7. It turns out that $\mathrm{S}^{r}$ is a special case of Chellas' Condition Logic. The modality ' $[\varphi]$ ' represents the knowledge state under the hypothesis $\varphi$. The formula $[\varphi] \psi$ states that 'under the hypothesis $\varphi$, the agent knows $\psi$ '. If $\varphi$ happens to be true at the current world and the agent knows that $\varphi$ implies $\psi$, then the agent knows $\psi$; otherwise, i.e., if $\varphi$ does not hold at the current world, the agent knows only what it would know anyway, i.e. without any assumptions. For instance, consider a simple dice game, where the game is won if, and only if, a three or a six has been rolled. The formula ' $[T]$ (three $\vee \operatorname{six} \leftrightarrow$ win)' states that the agent knows this rule. The parameter ' $T$ ' of the box-modality stands for the fact that no hypothesis is being adopted by the agent. Suppose that the dice is rolled under a cup, so that the rolled number of points is concealed from the agent. Consequently, as long as the dice remains concealed, the agent does not know whether or not the game is won. This can be described by the formula ' $\neg[\top]$ win'. However, the agent knows that the game is won under the hypothesis that a six has been rolled: '[ six] win'. We can distinguish two situations: one, where the hypothesis is correct, i.e., a six has been rolled; and another one, where it is false, i.e., the dice shows a number between one to five. In the former situation, the game is won and we have that the formula holds true. In case the hypothesis is in fact wrong, the formula is not necessarily true. Irrespective of the hypotheses held by the agent, the game may still be won provided that a three has been rolled. In all other cases, the game is lost. This is different to ordinary implications, which are true whenever the premise is false or the consequent is true.

Sentences in English of the form "If $A$, then $B . "$ are called conditional sentences. Here, $A$ is called the antecedent (or condition) and $B$ the consequent. Conditional sentences are traditionally put into different categories (according to mood or tense) such as indicative/subjunctive or factual/counterfactual. However, there is much disagreement on the logical theory of conditional sentences (in particular that of defeasible conditionals). One logical formalization is Conditional Logic, which essentially is Propositional Logic extended with a binary operator ' $\Rightarrow$ ' standing for conditionality. Several readings of ' $\Rightarrow$ ' were proposed, among them counterfactual conditional, non-monotonic consequence relation, normality and belief revision. Historically several logical accounts of conditionals have been suggested, among them Stalnaker 22, Lewis [16] and Chellas 7].

The logic $\mathrm{S}^{r}$ rejects the common assumption that logics allow to conclude anything from false premises. We borrow the term 'explosive' from Paraconsistent Logic, but we refer to conditional operators instead of the logical consequence relation. We say that a conditional operator ' $X$ ' is explosive if the conditional $\varphi X \psi$ holds for all conclusions $\psi$ whenever the antecedent $\varphi$ is false. In this sense, implication of Classical Logic and even of Intuitionistic Logic is explosive, so is the conditional operator ' $\Rightarrow$ ' of Conditional Logic [16, 22. On the other hand, the relativized necessity of our logic, which is a special case of Chellas' conditional operator [7], [8], is not explosive. We have that $[\perp] \psi$ is true iff $\psi$ is universally true. The consequence relation of $\mathrm{S}^{r}$ is not paraconsistent.

Epistemic logic traditionally describes the knowledge state of agents at a point in time. To be able to describe the evolution of knowledge over time, we can either combine epistemic logic with a temporal logic, or add dynamic operators for knowledge-changing actions such as communication. The latter approach is followed in the family of Dynamic Epistemic Logics 31. A basic DEL is Public Announcement Logic (PAL) [19] which is the extension of the basic epistemic logic S5 with an operator '[.]' parameterised with a formula expressing the announcement. A formula of the form $[\varphi] \psi$ states that $\psi$ holds after $\varphi$ has been truthfully announced (by someone) to every agent in the system simultaneously. After the announcement, $\varphi$ is incorporated in the knowledge state of every agent, i.e. $\varphi$ becomes common knowledge. This is achieved by employing an update semantics, which cuts off the model all worlds in which the announcement does not hold.

Our relative necessity operator '[.]' bears an interesting relationship to Provability Logic [6]. This is a modal logic, where the modality is considered to capture the metamathematical concept of 'a proposition being provable in some arithmetical theory'. An important logic in this context is the Gödel-Löb system GL as it characterises provability in Peano Arithmetic. A recent line of research is to modify GL and study the effects of these modifications w.r.t. provability [10]. For instance, [10] introduces three variants 
of the modality in GL and studies their algebraic semantics. Here is where the connection to this paper turns up as one of the modified modalities corresponds syntactically to the relative necessity operator '[.]' considered in this paper. To be precise, the definition of the modal operator called 'Modest Enrichment (Type B)' in [10] equals Axiom (R) $[\varphi] \psi \leftrightarrow \square \psi \vee \varphi \wedge \square(\varphi \rightarrow \psi)$, which we introduce below. In this paper, however, we do not investigate further the relationship to Provability Logic.

In this paper, we present an axiomatization of $\mathrm{S}^{r}$ and its extension with the common knowledge operator [25] as well as distributed knowledge operator 26] in Kripke and topological semantics. The paper is organized as follows. In the next section, we briefly recall basic definitions on modal logic, including a modal language and the Kripke semantics, and its standard translation into first-order logic. We review a technique for obtaining Kripke completeness results for certain extensions of a modal logic. we also review a technique for obtaining topological completeness from Kripke completeness based on the Alexandroff connection between preorders and Alexandroff spaces. In Section 3 , we recall the logic $\mathrm{S}^{r}$. In Section 4, we show that the technique from section 2 is applicable to $\mathrm{S}^{2} \mathrm{C}^{r}$ and thus prove the completeness results. Finally, the extension of $\mathrm{S}^{r}$ with modalities for distributed hypotheses is discussed in Section 5. We show how knowledge of an agent can be represented as distributed hypotheses, where the agents' knowledge corresponds to any system between S4 and S5 some constraints. We prove Kripke completeness and topological completeness results for the logic S5D ${ }^{r}$. Finally, the paper closes with a summary and outlook in Section 6

\section{Preliminaries}

In this section, we briefly review modal logic, cf. [5]. Moreover, we introduce two techniques for obtaining completeness results: one for obtaining Kripke completeness for the extensions/enrichments of Kripke complete logics, and the other for obtaining topological completeness from Kripke completeness. The first technique is introduced in detail in 24, whereas the second is well known in the literature 4, 3.

Let $\langle\Pi, M\rangle$ be a signature consisting of countable sets $\Pi$ and $M$ of symbols for propositions and modalities, respectively. The propositional modal language $\mathcal{L}$ for this signature consists of formulas $\varphi$ that are built up inductively according to the grammar:

$$
\varphi:=p|\neg \varphi| \varphi \wedge \varphi \mid \square m \varphi
$$

where $p$ ranges over proposition symbols in $\Pi$ and $m$ over modality symbols in $M$. The logical symbols ' $T$ ', and ' $\perp$ ', and the additional connectives such as ' $\vee$ ', ' $\rightarrow$ ' and ' $\leftrightarrow$ ' and the dual modalities ' $\nabla_{m}$ ' with $m \in M$ are defined as usual, i.e.: $\top:=p \vee \neg p$ for some atomic proposition $p ; \perp:=\neg \top ; \varphi \vee \psi:=\neg(\neg \varphi \wedge \neg \psi)$; $\varphi \rightarrow \psi:=\neg \varphi \vee \psi ; \varphi \leftrightarrow \psi:=(\varphi \rightarrow \psi) \wedge(\psi \rightarrow \varphi)$; and $\diamond_{m} \varphi:=\neg \square_{m} \neg \varphi$.

A subset $L$ of the propositional modal language $\mathcal{L}$ is a modal logic iff it contains all propositional tautologies, is closed under substitution, modus ponens and modal replacement (MREP) $\frac{p \leftrightarrow q}{\square_{m} p \leftrightarrow \square_{m} q}$, for $m \in M$. The modal logic $L$ is called normal if it contains the formulas (K) $\square_{m}(p \rightarrow q) \rightarrow\left(\square_{m} p \rightarrow \square_{m} q\right)$ and are closed under (NEC) $\frac{p}{\square_{m p}}$. The smallest normal modal logic is commonly denoted with $\mathrm{K}$.

\subsection{Kripke Semantics}

The relational semantics for the propositional modal language $\mathcal{L}$ is based on Kripke structures for the signature $\langle\Pi, M\rangle$ of $\mathcal{L}$. Formally, an $M$-frame (or Kripke frame) is a tuple $\mathfrak{F}=\left(W,\left\{R_{m}\right\}_{m \in M}\right.$ ), where $W$ is a non-empty set of worlds and $R_{m} \subseteq W^{2}$ a binary relation over $W$, for every $m \in M$. A Kripke model for $\langle\Pi, M\rangle$ is a pair $\mathfrak{M}=(\mathfrak{F}, V)$ consisting of a Kripke frame $\mathfrak{F}=\left(W,\left\{R_{m}\right\}_{m \in M}\right)$ together with a valuation function $V: \Pi \rightarrow 2^{W}$ assigning to every proposition $p$ in $\Pi$ a set $V(p)$ of worlds. A Kripke model $\mathfrak{M}=(\mathfrak{F}, V)$ is said to be based on the frame $\mathfrak{F}$.

An interpretation of formulas from $\mathcal{L}$ is given by means of a satisfaction relation ' $\models$ ', which is a binary relation between pointed models and formulas. A pointed model is a pair $\langle\mathfrak{M}, w\rangle$, where $\mathfrak{M}=\left(W,\left\{R_{m}\right\}_{m \in M}, V\right)$ is a Kripke model and $w$ a world from $W$. The satisfaction relation is defined inductively on the structure of formulas $\varphi$ as:

- $\langle\mathfrak{M}, w\rangle \models p$ iff $w \in V(p)$;

• $\langle\mathfrak{M}, w\rangle \models \neg \psi$ iff $\langle\mathfrak{M}, w\rangle \not \models \psi ;$ 
- $\langle\mathfrak{M}, w\rangle \mid=\psi \wedge \chi$ iff $\langle\mathfrak{M}, w\rangle \models \psi$ and $\langle\mathfrak{M}, w\rangle \models \chi ;$

- $\langle\mathfrak{M}, w\rangle=\square_{m} \psi$ iff for all $v \in W$ with $(w, v) \in R_{m},\langle\mathfrak{M}, v\rangle \models \psi$.

A formula $\varphi$ is said to be true at $w$ in $\mathfrak{M}$ iff $\langle\mathfrak{M}, w\rangle \models \varphi ; \varphi$ is satisfiable iff there is a pointed model $\langle\mathfrak{M}, w\rangle$ at which it is true; $\varphi$ is valid in $\mathfrak{M}$ (written ' $\mathfrak{M}=\varphi^{\prime}$ ) iff $\langle\mathfrak{M}, w\rangle=\varphi$ for all $w$ in $\mathfrak{M}$; $\varphi$ is valid on $\mathfrak{F}$ (written ' $\mathfrak{F}=\varphi$ ') iff $\varphi$ is valid in all models based on $\mathfrak{F}$; and $\varphi$ is valid in the class $\mathcal{C}$ of Kripke frames (written ' $=\mathcal{C} \varphi^{\prime}$ ) iff it is valid in every Kripke frame from $\mathcal{C}$.

The set of $\mathcal{L}$-formulas that are valid in a class $\mathcal{C}$ of Kripe frames is called the $\mathcal{L}$-theory $\operatorname{Th}_{\mathcal{L}}(\mathcal{C})$ of $\mathcal{C}$, i.e.:

$$
\operatorname{Th}_{\mathcal{L}}(\mathcal{C}):=\{\varphi \in \mathcal{L} \mid \text { for every } \mathfrak{F} \text { from } \mathcal{C}, \varphi \text { is valid in } \mathfrak{F}\} .
$$

A modal logic $L$ is said to be Kripke complete w.r.t. $\mathcal{C}$ iff $L \supseteq \operatorname{Th}_{\mathcal{L}}(\mathcal{C})$, and $L$ is said to be sound w.r.t. $\mathcal{C}$ iff $L \subseteq \operatorname{Th}_{\mathcal{L}}(\mathcal{C})$.

\subsection{Standard Translation}

The relationship to first-order logic is made precise by the so-called standard translation $\mathrm{ST}(\cdot)$, which assigns to a modal formula $\varphi$ a corresponding first-order formula $\operatorname{ST}_{x}(\varphi)$ with one free variable $x$. The signature of the first-order language contains unary predicate symbols $P$ and binary predicate symbols $R_{m}$, one $P$ for every $p \in \Pi$ and one $R_{m}$ for every $m \in M$. The translation function $\operatorname{ST}(\cdot)$ is inductively defined as follows:

$$
\begin{aligned}
\operatorname{ST}_{x}(p) & :=P(x) \\
\operatorname{ST}_{x}(\neg \varphi) & :=\neg \operatorname{ST}_{x}(\varphi) \\
\operatorname{ST}_{x}(\varphi \wedge \psi) & :=\operatorname{ST}_{x}(\varphi) \wedge \operatorname{ST}_{x}(\psi) \\
\operatorname{ST}_{x}\left(\square_{m} \varphi\right) & :=\forall y\left(R_{m}(x, y) \rightarrow \operatorname{ST}_{y}(\varphi)\right)
\end{aligned}
$$

where $y$ is a fresh variable for every occurrence of a box-modality.

A Kripke structure $\mathfrak{M}=\left(W,\left\{R_{m}\right\}_{m \in M}, V\right)$ for $\langle\Pi, M\rangle$ can be seen as a first-order structure interpreting the formula $\operatorname{ST}_{x}(\varphi)$. While a predicate symbol $R_{m}$ is interpreted using the binary relation $R_{m}$ over $W$ that is interpreting the modality $m$ in $M$, a predicate symbol $P$ is interpreted as the subset $V(p)$ of $W$, where $p$ is the proposition symbol from $\Pi$ that corresponds to $P$. Neither constants nor function symbols are introduced by the standard translation. In the first-order structure $\mathfrak{M}$, however, we introduce a dedicated constant $c_{w}$ for every world $w \in W$ and we interpret $c_{w}$ as $w$. At the level of pointed models $\langle\mathfrak{M}, w\rangle$, the relationship between $\varphi$ and $\operatorname{ST}_{x}(\varphi)$ is $\operatorname{such}$ that:

$$
\langle\mathfrak{M}, w\rangle \models \varphi \text { iff } \mathfrak{M} \models \operatorname{ST}_{x}(\varphi)\left[x \mapsto c_{w}\right]
$$

where $\left[x \mapsto c_{w}\right]$ substitutes every occurrence of the free variable $x$ in $\operatorname{ST}_{x}(\varphi)$ with the constant $c_{w}$. Note that $\operatorname{ST}_{x}(\varphi)\left[x \mapsto c_{w}\right]$ is a sentence, i.e. a first-order formula without free variables.

When considering the notion of validity on frames $\mathfrak{F}$, we have that $\varphi$ corresponds to the monadic second-order formula $\forall \vec{P} \forall x \operatorname{ST}_{x}(\varphi)$ as follows:

$$
\mathfrak{F} \models \varphi(\vec{p}) \text { iff } \mathfrak{F}=\forall \vec{P} \forall x \operatorname{ST}_{x}(\varphi)
$$

where $\vec{p}$ are the propositions from $\Pi$ that occur in $\varphi$, and $\vec{P}$ are the corresponding unary predicates.

\subsection{Completeness by Modal Definitions}

In 24] we introduced a technique on how to obtain Kripke completeness w.r.t. a specific class of Kripke structures for certain extensions of complete modal logics. We apply this technique to extensions of the modal logic S5.

By extending a modal logic $L$ with a formula $\varphi$ we mean obtaining a modal logic $L^{\prime}$ as a set of formulas that is minimal w.r.t. $\subseteq$, that contains all tautologies over the symbols for propositions occurring in $L \cup\{\varphi\}$, that contains all formulas from $L \cup\{\varphi\}$ and that is closed under substitution, modus-ponens and modal replacement. Clearly $L \cup\{\varphi\}$ is not necessarily a modal logic. Moreover, an extension of a modal logic that is Kripke complete w.r.t. a class $\mathcal{C}$ of models is not necessarily complete w.r.t. $\mathcal{C}$ itself nor 
any other class of models. We are interested in studying formulas of a specific form (modal definitions) that, when used to extend a modal logic, yield a modal logic that is complete w.r.t. a specific class of models. Let $\mathcal{L}$ be a propositional modal language over the signature $\langle\Pi, M\rangle$. Let $\varphi(\vec{p})$ be a formula in $\mathcal{L}$, where $\vec{p}$ are the propositions occurring in $\varphi$. Let ' + ' be a fresh symbol for a unary modality not in $M$, and $\boxplus$ the box-version of this modality. A modal definition in $\mathcal{L}$ is a formula of the form

$$
\boxplus p \leftrightarrow \varphi(\vec{p})
$$

where $\vec{p}$ contains $p$. The box-modality $\boxplus$ is defined in terms of a modal formula in which $\boxplus$ does not occur. Notice that the modal definition $\boxplus p \leftrightarrow \varphi(\vec{p})$ itself is a formula in the propositional modal language over the extended signature $\langle\Pi, M \cup\{+\}\rangle$. We only consider + to be a unary modality symbol. Moreover, we will only consider the modal definitions for the box-version of + . The results for the dual modality can be obtained in a similar way.

In this paper, we only consider modal definitions $\boxplus p \leftrightarrow \varphi(\vec{p})$, where the box-modality $\boxplus$ does not occur in $\varphi(\vec{p})$.

A modal definition is interpreted in models $\mathfrak{M}=(\mathfrak{F}, V)$ that are based on $M \cup\{+\}$-frames $\mathfrak{F}=$ $\left(W,\left\{R_{m}\right\}_{m \in M} \cup\left\{R_{+}\right\}\right)$, i.e., frames that are extended with a binary relation $R_{+}$to interpret the new box-modality $\boxplus$. The semantics of $\boxplus$ can be defined in the usual way as for any other box-modality:

- $\langle\mathfrak{M}, w\rangle \models \boxplus \psi$ iff for all $v \in W$ with $(w, v) \in R_{+}$, it holds that $\langle\mathfrak{M}, v\rangle \models \psi$.

We want to interpret $\boxplus$ as specified in the modal logic $L^{\prime}$ obtained from the modal logic $L$ extended with a modal definition of $\boxplus$. To this end, we have to confine ourselves to the models from $\mathcal{C}\left(L^{\prime}\right)$, i.e., all models from $\mathcal{K}_{\langle\Pi, M \cup\{+\}\rangle}$ in which all formulas of $L^{\prime}$ are valid. It is now interesting to investigate the relationship between the modal definition of $\boxplus$ and the properties of the relation $R_{+}$in the models from $\mathcal{C}\left(L^{\prime}\right)$.

Example 1. Let $\mathcal{L}$ be a propositional modal language over a signature $\langle\Pi, M\rangle$. Additionally, let ' + ' be a fresh symbol for a modality not in $M$. Finally, let $L \subseteq \mathcal{L}$ be a modal logic.

The modal definition $\alpha_{1}=\boxplus p \leftrightarrow p$ yields that $R_{+}$is the identity relation. This can be seen as follows. Obtain the modal logic $L_{1}$ by extending $L$ with $\alpha_{1}$. One can see that class of frames for $L_{1}$ is the class of frames for $L$ extended with the relation $R_{+}$being the identity relation.

Another simple example of a modal definition is $\boxplus p \leftrightarrow \square_{m} p$, for some $m \in M$. Here we have that $R_{+}$ equals $R_{m}$ in every model. Consider two more examples: $\boxplus p \leftrightarrow p \vee \neg p$ and $\boxplus p \leftrightarrow p \wedge \neg p$. In the former case, $R_{+}$is the empty relation, whereas in the latter case the modal definition does not yield any relation.

As the examples show, not all modal definitions yield a relational semantics for the logic extended with the newly defined modality. Taking the standard translation of a formula $\varphi$ that is used in a definition $\boxplus p \leftrightarrow \varphi(\vec{p})$ results in the second-order formula $\forall \vec{P} \forall x \operatorname{ST}_{x}(\varphi)$, where the predicates in $\vec{P}$ correspond to the propositional variables in $\vec{p}$. We are interested in elementary formulas, i.e., those formulas $\varphi$ for which there exists a first-order formula that is equivalent to the second-order formula $\forall \vec{P} \forall x \operatorname{ST}_{x}(\varphi)$, that additionally yield a relational semantics for the new modality + . It is a non-trivial problem to give a syntactic characterization of such formulas $\varphi$ that are suitable for defining fresh modalities.

To start tackling this problem, we introduce the notion of a 'relational modal definition'.

Definition 1. Let $\mathcal{L}$ be a propositional modal language over the signature $\langle\Pi, M\rangle$. Let $\varphi\left(p, p_{1}, \ldots, p_{n}\right)$ with $n \geq 0$ be a formula in $\mathcal{L}$, where $p, p_{1}, \ldots, p_{n}$ are the propositions occurring in $\varphi$. Let ' + ' be a fresh symbol for a unary modality not in $M$, and $\boxplus$ the box-version of this modality.

A modal definition $\boxplus p \leftrightarrow \varphi\left(p, p_{1}, \ldots, p_{n}\right)$ is called a relational modal definition if there exists a firstorder formula $\Psi_{+}(x, y)$ with two free variables $x$ and $y$ using only predicates that occur in $\operatorname{ST}_{x}\left(\varphi\left(p, p_{1}\right.\right.$, $\left.\ldots, p_{n}\right)$ ) such that for every $\psi \in \mathcal{L}$, it holds that for all pointed models $\langle\mathfrak{M}, w\rangle, \mathfrak{M} \models(\forall y)\left(\Psi_{+}(x, y) \rightarrow\right.$ $\left.\operatorname{ST}_{y}(\psi)\right)\left[x \mapsto c_{w}\right]$ iff $\mathfrak{M}=\operatorname{ST}_{x}\left(\varphi\left(\psi, p_{1}, \ldots, p_{n}\right)\right)\left[x \mapsto c_{w}\right]$.

Example 2. Let us consider modal logic $K$ extended with a new modality $\boxplus$. A formula $\boxplus p \leftrightarrow \square p \wedge p$ is a relational modal definition. Indeed, for every pointed model $\langle\mathfrak{M}, w\rangle$, it holds that $\mathfrak{M}=((\forall y)(x R y \rightarrow$ $P(y)) \wedge P(x))\left[x \mapsto c_{w}\right]$ iff $(\forall y)(\Psi(x, y) \rightarrow P(y))\left[x \mapsto c_{w}\right]$, where $\Psi(x, y)$ is the formula $(x R y) \vee(x=y)$.

We note that elementarity is neither a sufficient nor a necessary condition for modal formulas being suitable for a relational modal definition; see, e.g., the reduction axiom for $\mathrm{S}^{r}$ in the following section which yields a relational modal definition despite it being non-elementary. 
Let $\Psi_{+}(x, y)$ be the first-order formula with two free variables $x$ and $y$ corresponding to a relational modal definition. Given a model $\mathfrak{M}=(\mathfrak{F}, V)$ with $\mathfrak{F}=\left(W,\left\{R_{m}\right\}_{m \in M}\right)$, we uniquely construct the model $\mathfrak{M}_{+}=\left(\mathfrak{F}_{+}, V\right)$, where the underlying frame $\mathfrak{F}_{+}$is obtained from $\mathfrak{F}$ by adding the binary relation $R_{+} \subseteq W \times W$ defined as:

$$
(v, w) \in R_{+} \text {iff } \mathfrak{M} \models \Psi_{+}(x, y)\left[x \mapsto c_{v}, y \mapsto c_{w}\right]
$$

For a class $\mathcal{C}$ of models, we denote with $\mathcal{C}_{+}$the class consisting of the models $\mathfrak{M}_{+}$, where $\mathfrak{M}$ ranges over the models in $\mathcal{C}$.

Formulas from the extended language $\mathcal{L}_{+}$can be translated to formulas in $\mathcal{L}$ in a straightforward way.

Definition 2. Let $\mathcal{L}$ and $\mathcal{L}_{+}$be propositional modal languages over the signatures $\langle\Pi, M\rangle$ and $\langle\Pi, M \cup$ $\{+\}\rangle$, respectively, where + is a fresh unary modality not in $M$. The translation function ${ }^{*}: \mathcal{L}^{+} \rightarrow \mathcal{L}$ for the modal definition $\boxplus p \leftrightarrow \varphi_{+}\left(p, p_{1}, \ldots, p_{n}\right)$ is inductively defined as follows, where $m$ ranges over $M$ :

$$
\begin{aligned}
p^{*} & :=p \\
(\varphi \vee \psi)^{*} & :=\varphi^{*} \vee \psi^{*} \\
(\neg \varphi)^{*} & :=\neg \varphi^{*} \\
\left(\square_{m} \varphi\right)^{*} & :=\square_{m} \varphi^{*} \\
(\boxplus \psi)^{*} & :=\varphi_{+}\left(\psi^{*}, p_{1}, \ldots, p_{n}\right)
\end{aligned}
$$

The following theorem shows the intended completeness technique.

Theorem 1 ([24]). Let $\mathcal{L}$ and $\mathcal{L}_{+}$be propositional modal languages over the signatures $\langle\Pi, M\rangle$ and $\langle\Pi, M \cup$ $\{+\}\rangle$, respectively, where + is a fresh unary modality not in $M$. Let $L \subseteq \mathcal{L}$ be a normal modal logic that is sound and complete w.r.t. a class $\mathcal{F}$ of Kripke frames. Obtain $L_{+} \subseteq \mathcal{L}_{+}$from $L$ by adding a relational modal definition $\boxplus p \leftrightarrow \varphi\left(p_{1}, \ldots, p_{n}\right)$ as an only axiom schema for $\boxplus$. Then the logic $L_{+}$is sound and complete w.r.t. the class $\mathcal{F}_{+}$.

\subsection{Topological Semantics}

In [17 McKinsey\&Tarski introduced topological semantics of modal logic. A pair $(X, \tau)$ is called a topological space iff $X$ is a set and $\tau$ is a collection of subsets of $X$ with the following properties: (i) $X, \emptyset \in \tau$; (ii) $A, B \in \tau$ implies $A \cap B \in \tau$; and (iii) $A_{i} \in \tau$, for all $i \in I$, implies $\bigcup_{i \in I} A_{i} \in \tau$, for some index set $I$. Condition (ii) states that $\tau$ contains every finite intersection of its members, whereas (iii) states that $\tau$ contains every finite or infinite union of its members. Elements of a topological space $\tau$ are called open sets of $\tau$. Closed sets are defined as complements of open sets. A topological space $(X, \tau)$ is called an Alexandroff space iff an arbitrary (finite or infinite) intersection of open sets is open, i.e., for some index set $I$ it holds that $A_{i} \in \tau$, for all $i \in I$, implies $\bigcap_{i \in I} A_{i} \in \tau$. For a topological space $(X, \tau)$,

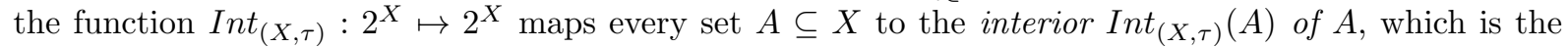
maximal open set included in $A$, i.e., the set $\operatorname{Int}_{(X, \tau)}(A)$ is a subset of $A$ that is open w.r.t. $\tau$, and it is maximal w.r.t. $\subseteq$ among the open sets w.r.t. $\tau$ that are subsets of $A$. A topological space $(X, \tau)$ uniquely determines the function $\operatorname{Int}_{(X, \tau)}$. We drop $(X, \tau)$ from the subscript of $\operatorname{Int}_{(X, \tau)}$ if it is clear from the context. An interior operator satisfies the following conditions, also known as Kuratowski axioms: (i) $\operatorname{Int}(X)=X$; (ii) $\operatorname{Int}(A) \subseteq A$; (iii) $\operatorname{Int}(\operatorname{Int}(A))=\operatorname{Int}(A)$; and (iv) $\operatorname{Int}(A \cap B)=\operatorname{Int}(A) \cap \operatorname{Int}(B)$.

An interpretation of formulas from $\mathcal{L}$ on topological spaces is given by means of a satisfaction relation ' $=$ ', which is a binary relation between pointed topological models and formulas. A pointed topological model is a pair $\langle\mathfrak{T}, x\rangle$, where $\mathfrak{T}=\left(X,\left\{\tau_{m}\right\}_{m \in M}, V\right)$ is a topological space with a topology $\tau_{m}$ for every $m \in M$ extended with a valuation $V$, and $x$ is an element of the set $X$. The satisfaction relation is defined inductively on the structure of formulas $\varphi$ as:

- $\langle\mathfrak{T}, x\rangle \models p$ iff $x \in V(p)$;

- $\langle\mathfrak{T}, x\rangle=\neg \psi$ iff $\langle\mathfrak{T}, x\rangle \not \mid \psi$;

• $\langle\mathfrak{T}, x\rangle \models \psi \wedge \chi$ iff $\langle\mathfrak{T}, x\rangle \models \psi$ and $\langle\mathfrak{T}, x\rangle \models \chi$;

- $\langle\mathfrak{T}, x\rangle \models \square_{m} \psi$ iff $x \in \operatorname{Int}_{m}(\{y \mid\langle\mathfrak{T}, y\rangle \models \psi\})$, 
where $I n t_{m}$ is the interior operator of the topological space $\left(X, \tau_{m}\right)$. A formula $\varphi$ is said to be true at $x$ in $\mathfrak{T}$ iff $\langle\mathfrak{T}, x\rangle \models \varphi ; \varphi$ is satisfiable iff there is a pointed topological model $\langle\mathfrak{T}, x\rangle$ at which it is true; $\varphi$ is valid in $\mathfrak{T}$ (written ' $\mathfrak{T}=\varphi$ ') iff $\langle\mathfrak{T}, x\rangle=\varphi$ for all $x$ in $\mathfrak{T} ; \varphi$ is valid in the topological space $\left(X,\left\{\tau_{m}\right\}_{m \in M}\right)$ (written ' $\left.\left(X,\left\{\tau_{m}\right\}_{m \in M}\right)=\varphi^{\prime}\right)$ iff $\varphi$ is valid in all topological models based on $\left(X,\left\{\tau_{m}\right\}_{m \in M}\right)$; and $\varphi$ is valid in the class $T$ of topological spaces (written ' ${ }^{\prime}=_{T} \varphi^{\prime}$ ) iff it is valid in every topological space from $T$.

A modal logic $L \subseteq \mathcal{L}$ is said to be topologically complete w.r.t. $T$ iff $L \supseteq \operatorname{Th}_{\mathcal{L}}(T)$, and $L$ is said to be sound w.r.t. $T$ iff $L \subseteq \operatorname{Th}_{\mathcal{L}}(T)$. The notion of a theory $\operatorname{Th}_{\mathcal{L}}(\cdot)$ generalizes from Kripke structures to topological spaces in a straightforward way.

\subsection{Completeness by Alexandroff connection}

A well-known technique for obtaining topological completeness from Kripke completeness utilises the relationship between preorders and Alexandroff topologies.

Proposition 1 ([10]). There is a one-to-one correspondence between preorders and Alexandroff spaces on a given set $X$. Moreover, if we consider the lattice Pre $(X)$ of all preorders on a set $X$ and the lattice of all Alexandroff topologies $\operatorname{Top}_{A l}(X)$ on the same set $X$, the two lattices are unti-isomorphic.

To illustrate the correspondence, we first introduce the downset operator. For a preorder $(X, R)$, the downset operator $R^{-1}$ is defined as follows: For every $A \subseteq X$, we set $R^{-1}(A):=\{x \mid(\exists y)(y \in A \wedge x R y)\}$. Now, if we are given a preorder $(X, R)$, it is possible to prove that the downset operator $R^{-1}$ satisfies all the properties of the topological closure operator. Hence, we obtain a topological space $\left(X, \tau_{R}\right)$, where $\tau_{R}$ is the topology obtained from the closure operator $R^{-1}$. Conversely, every Alexandroff space $(X, \tau)$ can be associated with a preorder $\left(X, R_{\tau}\right)$ such that $x R_{\tau} y$ iff $x \in C l(\{y\})$, where $C l$ denotes the closure operator of a topological space $(X, \tau)$. The set $A$ is open in $\left(X, \tau_{R}\right)$ iff $x \in A$ implies that the implication $(x R y \Rightarrow y \in A)$ holds for every $y \in X$.

If $R_{1}$ and $R_{2}$ are two preorders on $X$ and $\left(R_{1} \cup R_{2}\right)^{+}$is a transitive closure of the union of the two relations, then $\tau_{\left(R_{1} \cup R_{2}\right)^{+}} \cong \tau_{R_{1}} \cap \tau_{R_{2}}$ and $\tau_{\left(R_{1} \cap R_{2}\right)} \cong \tau_{R_{1}} \sqcup \tau_{R_{2}}$, where $\sqcup$ stands for the union topology of the two topologies.

Proposition 2 ([10]). The correspondence in the Proposition 1 preserves the truth of modal formulas. Formally, $(X, R, V), x=\varphi$ iff $\left(X, \tau_{R}, V\right), x=\varphi$, and $\left(X, R_{\tau}, V\right), x \models \varphi$ iff $(X, \tau, V), x=\varphi$.

\section{The Modal Logic S5}

In this section, we recall the multi-modal logic $\mathrm{S}^{r}$ from 23 together with the completeness result w.r.t. a particular class of models called basic structures. In addition, we provide the topological semantics of the $\mathrm{S}^{r}$ and prove topological completeness.

\subsection{Syntax}

The language of $\mathrm{S}^{r}$ is the language of propositional logic extended with modal operators parameterized with $\mathrm{S}^{r}$-formulas. Formally, this is done as follows. Let $\Pi$ be a countable set of propositions. Formulas $\varphi$ of the language $\mathcal{L}$ are defined inductively over $\Pi$ by the following grammar:

$$
\varphi, \psi::=p|\neg \varphi| \varphi \vee \mid[\varphi]_{K} \psi,
$$

where $p$ ranges over propositions in $\Pi$. The logical symbols ' $T$ ' and ' $\perp$ ', and additional operators such as ' $\wedge$ ', ' $\rightarrow$ ', ' $\leftrightarrow$ ', and the dual modalities ' $\langle\varphi\rangle_{K}$ ' are defined as usual.

\subsection{Relational semantics}

Modal formulas are commonly evaluated in models containing a binary relation over the domain, one for each modality in the modal language. In this case, however, every binary relation is determined by the valuation of the atomic propositions in the domain. Therefore, it is sufficient to consider models without relations, which we call basic structures. Formally, a basic structure $\mathfrak{M}$ is a tuple $\mathfrak{M}=(W, V)$, where $W$ is a non-empty set of worlds and $V: \Pi \rightarrow 2^{W}$ a valuation function mapping every atomic proposition $p$ 
to a set of worlds $V(p)$ at which it is true. The relations that are required to evaluate the modalities are defined alongside the satisfaction relation. But first we introduce an auxiliary notion, a binary operation ' $\otimes$ ' on sets yielding a binary relation. Let $X$ and $Y$ be two sets. Let $X \otimes Y$ be a binary relation over $X \cup Y$ such that

$$
X \otimes Y=X^{2} \cup(X \times Y) \cup Y^{2}
$$

We illustrate this notion with an example.

Example 3. Let $X=\left\{x_{1}, x_{2}\right\}$ and $Y=\left\{y_{1}, y_{2}, y_{3}\right\}$ be two sets. Then, according to Equation (1), X®Y is a binary relation over $X \cup Y$ that is composed of the relations $X^{2}, X \times Y$ and $Y^{2}$ by taking their union. It holds that $X^{2}=\left\{\left(x_{1}, x_{2}\right),\left(x_{2}, x_{1}\right)\right\} \cup i d(X), X \times Y=\left\{\left(x_{1}, y_{1}\right),\left(x_{1}, y_{2}\right),\left(x_{1}, y_{3}\right),\left(x_{2}, y_{1}\right),\left(x_{2}, y_{2}\right),\left(x_{2}, y_{3}\right)\right\}$ and $Y^{2}=\left\{\left(y_{1}, y_{2}\right),\left(y_{2}, y_{1}\right),\left(y_{1}, y_{3}\right),\left(y_{3}, y_{1}\right),\left(y_{2}, y_{3}\right),\left(y_{3}, y_{2}\right)\right\} \cup i d(Y)$. Then the relation $X \otimes Y=$ $X^{2} \cup(X \times Y) \cup Y^{2}$ contains two fully connected clusters $X^{2}$ and $Y^{2}$, and directed edges between every point in $X$ to every point in $Y$. Figure 1 below gives a graphical representation of $X \otimes Y$ (leaving out the reflexive and symmetric edges).

We are now ready to introduce the semantics of $\mathrm{S}^{r}$. It differs from the semantics of Public Announcement Logic [19, 31] in that the model does not change during the evaluation of formulas.

Let $\mathfrak{M}=(W, V)$ be a basic structure. The logical satisfaction relation ' $=$ ' is defined by induction on the structure of $\mathrm{S}^{r}$-formulas as follows: For all $p \in \Pi$ and all $\varphi, \psi \in \mathcal{L}$,

- $\langle\mathfrak{M}, w\rangle \models p$ iff $w \in V(p)$;

• $\langle\mathfrak{M}, w\rangle=\varphi \vee \psi$ iff $\langle\mathfrak{M}, w\rangle \models \varphi$ or $\langle\mathfrak{M}, w\rangle \models \psi$;

- $\langle\mathfrak{M}, w\rangle=\neg \varphi$ iff $\langle\mathfrak{M}, w\rangle \mid \vDash \varphi$;

- $\langle\mathfrak{M}, w\rangle \models[\varphi]_{K} \psi$ iff for all $v \in W$ with $(w, v) \in R_{\varphi}$, it holds that $\langle\mathfrak{M}, v\rangle \models \psi$;

where $R_{\varphi}=\left(W \backslash \llbracket \varphi \rrbracket_{\mathfrak{M}}\right) \otimes \llbracket \varphi \rrbracket_{\mathfrak{M}}$ as defined in Equation 11 and $\llbracket \varphi \rrbracket_{\mathfrak{M}}=\{x \in W \mid\langle\mathfrak{M}, w\rangle \models \varphi\}$ is the extension of $\varphi$ in $\mathfrak{M}$. We say that a $\mathrm{S}^{r}$-formula $\varphi$ is satisfiable if there is a model $\mathfrak{M}$ and a world $w$ in $\mathfrak{M}$ such that $\langle\mathfrak{M}, w\rangle=\varphi ; \varphi$ is valid in $\mathfrak{M}$ if $\langle\mathfrak{M}, w\rangle=\varphi$ for all $w$ in $\mathfrak{M}$; and $\varphi$ is valid if $\varphi$ is valid in all models. We will refer to the relation $R_{\varphi}$ as being determined by $\varphi$ and a model.

According to the semantics, a formula determines a binary relation in a model. The following proposition states the properties of such relations.

Proposition 3. Let $\varphi$ be an $S 5^{r}$-formula and let $\mathfrak{M}=(W, V)$ be a basic structure. Then, the relation $R_{\varphi}$ determined by $\varphi$ and $\mathfrak{M}$ (cf. Definition 3.2) is a one-step total preorder, i.e., $R_{\varphi}$ satisfies the following conditions:

- $R_{\varphi}$ is transitive: $\forall x y z\left(R_{\varphi}(x, y) \wedge R_{\varphi}(y, z) \rightarrow R_{\varphi}(x, z)\right)$;

- $R_{\varphi}$ is total: $\forall x y\left(R_{\varphi}(x, y) \vee R_{\varphi}(y, x)\right)$; and

- $R_{\varphi}$ is one-step: $\forall x y z\left(R_{\varphi}(x, y) \wedge \neg R_{\varphi}(y, x) \wedge R_{\varphi}(x, z) \rightarrow\left(z R_{\varphi} y\right)\right)$.

Instead of 'preorder' also the term 'quasiorder' is often used in the literature. Note that totality implies reflexivity and that a symmetric total preorder is an equivalence relation. The proposition is readily checked as any relation $R_{\varphi}$ in a model determined by $\varphi$ is defined using the operation ' $\otimes$ ', which always yields a so-called 'one-step total preorder'. As the domain of a model is non-empty, it contains at least one point and, thus, the smallest relation $R_{\varphi}$ is the edge of a single reflexive point.

Proposition 4. The relation $R_{\varphi}$ for every formula $\varphi \in S 5^{r}$ is characterized by the following condition: $R_{\varphi}(w, v)$ iff $w \in \llbracket \varphi \rrbracket$ implies that $v \in \llbracket \varphi \rrbracket$.

Figure 1 illustrates the relation $R_{\varphi}$ in a model $\mathfrak{M}$. The domain of $\mathfrak{M}$ is partitioned into two clusters, the worlds in each of which are fully connected (reflexive and symmetric edges within the clusters are not shown). Between the clusters there are outgoing directed edges from worlds in the cluster on the left- to worlds in the cluster on the right-hand side, but not vice versa. Revisit Example 3 to see in detail how $R_{\varphi}$ is computed (where $X=W \backslash \llbracket \varphi \rrbracket \mathfrak{M}$ and $Y=\llbracket \varphi \rrbracket_{\mathfrak{M}}$ ).

Consider the following example, which illustrates the effect that hypotheses can have on an agent's knowledge. 


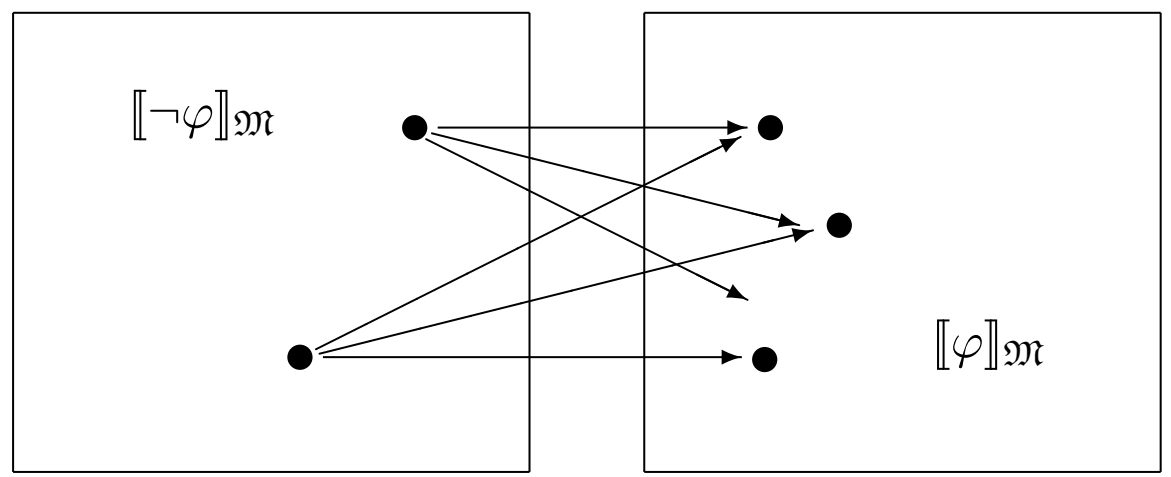

Figure 1: Model $\mathfrak{M}$ with Relation $R_{\varphi}$

Example 4. Let $\mathfrak{M}=(W, V)$ be a basic structure with $W=\{x, y\}, V\left(p_{h}\right)=V\left(p_{c}\right)=\{x\}$ and $V\left(p_{u}\right)=$ $\{x, y\}$. Intuitively, the three propositions $p_{h}, p_{c}$ and $p_{u}$ stand for hypothesis, conclusion and universal or already established knowledge, respectively. Then, $\left[p_{h}\right]_{K} p_{u}$ is true at $x$ and $y$ in $\mathfrak{M}$. In fact, we have that $\langle\mathfrak{M}, x\rangle \models[\varphi]_{K} p_{u}$ for every $S 5^{r}$-formula $\varphi$, because $p_{u}$ holds everywhere in $\mathfrak{M}$. But $\left[p_{h}\right]_{K} p_{c}$ holds only at $x$ and not at $y$, because $\langle\mathfrak{M}, x\rangle \models p_{h}$ and $p_{h}$ implies $p_{c}$ everywhere in $\mathfrak{M}$.

We conclude this section with a discussion on how $\mathrm{S} 5^{r}$ could possibly be used to reason about the knowledge of multiple agents; see, e.g., [11, [18] for standard references. Syntactically, $\mathrm{S}^{r}$ is a singleagent logic. That is, it does not provide us with syntactic markers to distinguish agents such as a different modality for each agent as in the modal epistemic logic $S 5_{n}$. Consequently, there is no way to distinguish different agents other than by what they know. In $S 5^{r}$ we can represent the individuality of agents in the hypothesis itself. For instance, in order to represent what the agents $a$ and $b$ know, we can use different hypotheses $p_{a}$ and $p_{b}$, which are atomic propositions labelling the states which the agents $a$ and $b$, respectively, consider possible. Thus $\left[p_{a}\right]_{K} \varphi$ states ' $a$ knows $\varphi$ ' and $\left[p_{b}\right]_{K} \psi$ states that ' $b$ knows $\psi$ '.

\subsection{Axiomatization}

We now present a sound and complete axiomatization of the logic $\mathrm{S}^{r}$ from [23]. The axiom system consists of all propositional tautologies and the following axioms:

$(\mathrm{K})[\top]_{K}(p \rightarrow q) \rightarrow\left([\top]_{K} p \rightarrow[\top]_{K} q\right)$

(Т) $[\top]_{K} p \rightarrow p$

(4) $[T]_{K} p \rightarrow[T]_{K}[\top]_{K} p$

(B) $p \rightarrow[\top]_{K} \neg[\top]_{K} \neg p$

(R) $[\varphi]_{K} p \leftrightarrow[\top]_{K} p \vee\left(\varphi \wedge[\top]_{K}(\varphi \rightarrow p)\right)$

The first four axioms are similar to the axioms known from the modal epistemic logic S5 characterizing any modality $[\varphi]_{K}$ in our logic $\mathrm{S}^{r}$ as epistemic operator that can be used to represent what is known under the hypothesis $\varphi$.

The axioms $(\mathrm{T}),(4)$, and (B) are for the modality $[T]_{K}$ only, whereas we need additional instances of the axioms $(\mathrm{K})$ and $(\mathrm{R})$, namely the ones for each modal parameter $\varphi$ (cf. Section 3.1). The reduction axiom (R) states that every modality $[\varphi]_{K}$ is definable in terms of the basic modal operator $[\top]_{K}$, which corresponds to the S5-box or the universal modality. As it was already mentioned in the introduction, Axiom (R) corresponds to the definition of the modal operator 'Modest Enrichment (Type B)' in [10].

Theorem 2 (23]). The system $S^{r}$ is sound and complete w.r.t. the class of basic structures. 


\subsection{Topological Semantics}

The topological semantics for $\mathrm{S}^{r}$ is provided by the pair $(X, \tau)$, where the set $X$ is equipped with the least topology $\tau$, i.e., the set of open sets $\tau=\{X, \emptyset\}$. We call such pairs topological structures.

A triple $(X, \tau, V)$, where $(X, \tau)$ is a topological structure and $V$ is a valuation function, is called a topological model. The formulas on topological models are evaluated as follows:

Let $\mathfrak{T}=(X, \tau, V)$ be a topological model. The logical satisfaction relation ' $\models$ ' is defined by induction on the structure of $\mathrm{S}^{r}$-formulas as follows: For all $p \in \Pi$ and all $\varphi, \psi \in \mathcal{L}$,

- $\langle\mathfrak{T}, w\rangle \models p$ iff $w \in V(p)$;

- $\langle\mathfrak{T}, w\rangle \models \varphi \vee \psi$ iff $\langle\mathfrak{T}, w\rangle \models \varphi$ or $\langle\mathfrak{T}, w\rangle \models \psi$;

- $\langle\mathfrak{T}, w\rangle \models \neg \varphi$ iff $\langle\mathfrak{T}, w\rangle \not=\varphi$;

- $\langle\mathfrak{T}, w\rangle \models[\varphi]_{K} \psi$ iff $w \in \operatorname{Int}_{\varphi}(\llbracket \psi \rrbracket)$, where $\operatorname{Int}_{\varphi}$ is the interior operator of the topology $\tau$ extended with one open set $\varphi \varphi \rrbracket$.

Let $\tau_{\varphi}$ denote the topology $\{\emptyset, \llbracket \varphi \rrbracket, X\}$ which is a refinement of a topology $\tau$ by the open set $\llbracket \varphi \rrbracket$. The main theorem of this section is a topological completeness result for the modal logic $\mathrm{S}^{r}$.

Lemma 1. The Alexandroff space $\tau_{R_{\varphi}}$ corresponding to the preorder $R_{\varphi}$ is exactly $\tau_{\varphi}$.

Proof. By the Alexandroff correspondence we know that the open sets of a space corresponding to the relation $R_{\varphi}$ are exactly up-sets of $R_{\varphi}$. By Proposition 4 , the relation $R_{\varphi}$ has only three up-sets $\emptyset, \llbracket \varphi \rrbracket$ and $X$. Hence the topology $\tau_{R_{\varphi}}$ is equal to $\tau_{\varphi}$.

The following lemma is essential for reducing the problem of topological completeness to Kripke completeness.

Lemma 2. For an arbitrary formula $\alpha$ from $S 5^{r}$ and an arbitrary structure $(W, V)$ the following holds: $(W, V), w=\alpha$ iff $(W, \tau, V), w \models \alpha$ where $\tau=\{\emptyset, W\}$ and $w$ is an arbitrary point from $W$.

Proof. The proof goes by induction on the complexity of formulas. The only non-trivial case is for modal formulas. For simplicity of presentation we won't write but assume that for the subformulas we by induction already have the desired iff condition. Let $\mathfrak{M}=(W, V)$ and $w \in W$ such that $\langle\mathfrak{M}, w\rangle \models[\varphi]_{K} \psi$. This is equivalent to $(\forall v)\left(R_{\varphi}(w, v) \Rightarrow v \models \psi\right)$. By Proposition 4 , we obtain that if $w \in \llbracket \varphi \rrbracket \Rightarrow v \in \llbracket \varphi \rrbracket$, then $v \models \psi$. We distinguish two cases. Case 1: $w \models \varphi$. For $v \in W$, if $v \models \varphi$, then the implication $w \in \llbracket \varphi \rrbracket \Rightarrow v \in \llbracket \varphi \rrbracket$ is satisfied. Hence, $v \models \psi$. This means that $\llbracket \varphi \rrbracket \subseteq \llbracket \psi \rrbracket$ and $w \in \llbracket \psi \rrbracket$. Additionally, by the properties of Alexandroff connection, we know that the upset $\llbracket \varphi \rrbracket$ is open in $\tau_{R_{\varphi}}$. By lemma 1 this means that $\llbracket \varphi \rrbracket$ is open in $\tau_{\varphi}$. Hence, $\operatorname{Int}_{\varphi}(\llbracket \psi \rrbracket) \supseteq \llbracket \varphi \rrbracket$. We conclude that $w \in \operatorname{Int}_{\varphi}(\llbracket \psi \rrbracket)$.

Case 2: $w \not \models \varphi$. In this case $w \in \llbracket \varphi \rrbracket \Rightarrow v \in \llbracket \varphi \rrbracket$ holds for an arbitrary $v \in W$. Hence, $\psi$ is valid in the entire model. This means that $\operatorname{Int}_{\varphi}(\llbracket \psi \rrbracket)=W$. Hence, $w \in \operatorname{Int}_{\varphi}(\llbracket \psi \rrbracket)$.

In either case, we obtain that $w \in \operatorname{Int}_{\varphi}(\llbracket \psi \rrbracket)$. By definition of satisfaction on topological models, this yields that $(W, \tau, V), w \models[\varphi]_{K} \psi$. The converse direction can be shown similarly.

Theorem 3. The logic $S 5^{r}$ is sound and complete w.r.t. the class of all topological structures.

Proof. Soundness can be proved by straightforward check. Completeness of the logic S5 ${ }^{r}$ w.r.t. topological structures immediately follows from the Kripke completeness result (Theorem 22 and lemma 2. Assume that $\varphi$ is not a $\mathrm{S}^{r}$ theorem. By Theorem 2 there is a basic structure $(W, V)$ which falsifies $\varphi$. And by lemma $2 \varphi$ is falsified on the topological structure $\mathfrak{T}=(W, \tau, V)$

\section{The Modal Logic S5C ${ }^{r}$}

Probably one of the most interesting examples in the direction of dynamic epistemic logic is provided by the notion of common knowledge. Common knowledge, originally defined by Lewis [15, has been extensively studied from various perspectives including philosophy [2], game theory [29] artificial intelligence [14] and Epistemic and modal logic [1. The usual definition of common knowledge of agents is 
given as an infinite conjunction of nested individual knowledge. For example in the case of two agents, say, agent 1 and agent 2 with corresponding knowledge operators $\square_{1}$ and $\square_{2}$, common knowledge that $p$ is formalized by an infinite formula of the form

$$
\varphi \wedge \square_{1} \varphi \wedge \square_{2} \varphi \wedge \square_{1} \square_{1} \varphi \wedge \square_{1} \square_{2} \varphi \wedge \square_{2} \square_{1} \varphi \wedge \square_{2} \square_{2} \varphi \wedge \square_{1} \square_{1} \square_{1} \varphi \ldots
$$

At the end of Section 3, we have explained that the logic $\mathrm{S}^{r}$ can be seen as a multi-agent logic if the labeling for the agents is given not by natural numbers but by formulas, i.e., the facts or hypotheses they have. With that in mind, it seems natural to extend the language with a modality for common knowledge. In this section, we extend $\mathrm{S}^{r}$ with modalities for common knowledge and prove Kripke completeness.

Definition 3 (Syntax of S5C ${ }^{r}$ ). Let $\Pi$ be a countable set of propositions. Formulas $\varphi$ of S5C ${ }^{r}$ are defined inductively over $\Pi$ by the following grammar:

$$
\varphi, \psi, \varphi_{i}::=p|\neg \varphi| \varphi \vee \quad\left|[\varphi]_{K} \quad\right|\left[\left\{\varphi_{1}, \ldots, \varphi_{n}\right\}\right]_{C} \psi,
$$

where $p$ ranges over atomic propositions in $\Pi$ and $n \geq 0$.

To improve readability, we index the modalities with ' $\mathrm{K}$ ' and ' $\mathrm{C}$ ' to indicate that they stand for knowledge and common knowledge, respectively. Moreover, we may omit the braces in $\left[\left\{\varphi_{1}, \ldots, \varphi_{n}\right\}\right]_{C} \psi$ and simply write $\left[\varphi_{1}, \ldots, \varphi_{n}\right]_{C} \psi$ instead.

Formulas of $\mathrm{S}^{2} \mathrm{C}^{r}$ are evaluated in basic structures as well. A modality $[\Phi]_{C}$ is a necessity depending on the formulas in the set $\Phi$. The semantics of $[\Phi]_{C}$ is based on the relations $R_{\varphi}$ with $\varphi \in \Phi$ as follows.

Definition 4 (Semantics of $\left.\mathrm{S}^{2} \mathrm{C}^{r}\right)$. Let $\mathfrak{M}=(W, V)$ be a basic structure. The satisfaction relation ' $=$, and the relations $R_{\varphi}$ for formulas $\varphi$ of $S 5 C^{r}$ are defined as for $S 5^{r}$ but extended with the following clauses: For all $S 5 C^{r}$-formulas and all finite sets $\Phi$ of $S 5 C^{r}$-formulas,

- $\langle\mathfrak{M}, w\rangle \models[\Phi]_{C} \psi$ iff for all $v \in W$ with $(w, v) \in R_{\Phi}^{C}$, it holds that $\langle\mathfrak{M}, v\rangle \models \psi$,

where $R_{\Phi}^{C}=\left(\bigcup_{\varphi \in \Phi} R_{\varphi}\right)^{*}$ and $(\cdot)^{*}$ yields the reflexive and transitive closure of relation.

Due to the simple structure of one-step relations, it may seem the union $\bigcup_{\varphi \in \Phi} R_{\varphi}$ is itself transitive. The following example illustrates that this is not always the case.

Example 5. Let $\mathfrak{M}=(W, V)$ be a model, where $W=\{x, y, s, t\}, V(p)=\{t, y\}$ and $V(q)=\{x, t\}$. Then $R_{p}=\{s, x\} \otimes\{t, y\}$ and $R_{q}=\{s, y\} \otimes\{t, x\}$. Hence, we have that $\left(R_{p} \cup R_{q}\right)(t, y)$ and $\left(R_{p} \cup R_{q}\right)(y, s)$ but not $\left(R_{p} \cup R_{q}\right)(t, s)$; see Figure 2
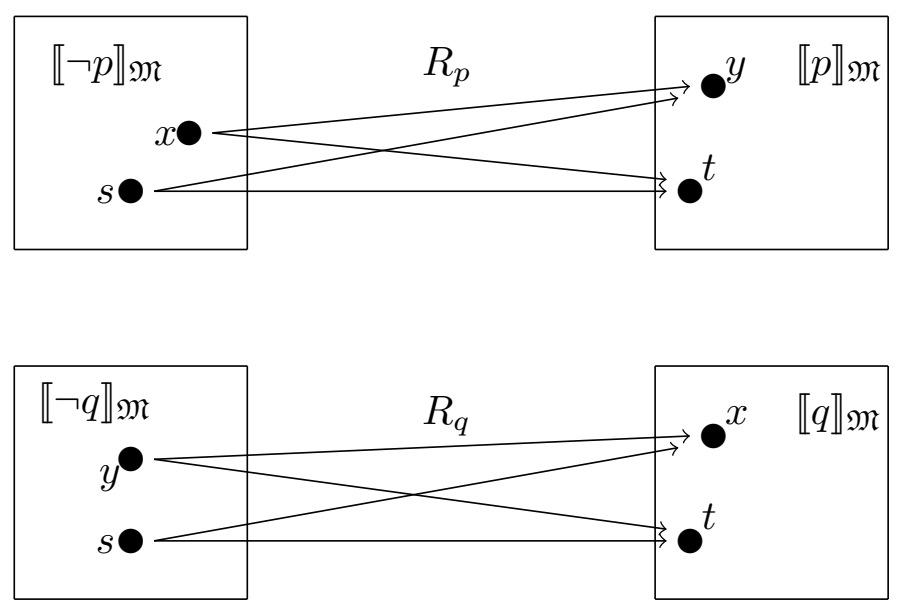

Figure 2: Model $\mathfrak{M}$ with Relations $R_{p}$ and $R_{q}$

The example also shows that the reflexive and transitive closure of the union relation $\bigcup_{\varphi \in \Phi} R_{\varphi}$ will be a cluster if for every pair $s$ and $t$ of worlds that are from different clusters of each relation $R_{\varphi}$ with $\varphi \in \Phi$, there are formulas $\theta$ and $\psi$ in $\Phi$ such that $\langle\mathfrak{M}, s\rangle \models \theta \wedge \neg \psi$ and $\langle\mathfrak{M}, t\rangle \models \neg \theta \wedge \psi$. 
In the remainder of the section, we focus on the case where $\Phi=\left\{\varphi_{1}, \varphi_{2}\right\}$. It can readily be seen that the results can be generalized to an arbitrary finite subset of formulas. The definition of the satisfaction relation boils down to the following:

- $\langle\mathfrak{M}, w\rangle \models[\Phi]_{C} \psi$ iff for all $v \in W: w\left(R_{\varphi_{1}} \cup R_{\varphi_{2}}\right)^{*} v$ implies that $\langle\mathfrak{M}, v\rangle \models \psi$.

The proof of the following proposition follows from the semantics of $[\Phi]_{C} \psi$ and properties of the transitive and reflexive closure of the relation.

Proposition 5. Let $\mathfrak{M}$ be a model and $w$ be a world in $\mathfrak{M}$. Then the following holds: $\langle\mathfrak{M}, w\rangle \models[\Phi]_{C}$ iff $\langle\mathfrak{M}, w\rangle=\psi \wedge\left[\varphi_{1}\right]_{K} \psi \wedge\left[\varphi_{2}\right]_{K} \psi \wedge\left[\varphi_{1}\right]_{K}\left[\varphi_{2}\right]_{K} \psi \wedge\left[\varphi_{2}\right]_{K}\left[\varphi_{1}\right]_{K} \psi \wedge\left[\varphi_{1}\right]_{K}\left[\varphi_{1}\right]_{K} \psi \wedge \cdots$.

The next proposition shows that the infinitary nature of the common knowledge operator could be captured by a formula of the language $\mathrm{S}^{r}$.

Proposition 6. Let $\mathfrak{M}$ be a model and $w$ be a world in $\mathfrak{M}$. Then the following holds: $\langle\mathfrak{M}, w\rangle \models$ $\left\langle\left\{\varphi_{1}, \varphi_{2}\right\}\right\rangle_{C} \psi$ iff $\langle\mathfrak{M}, w\rangle \models\left\langle\varphi_{1}\right\rangle_{K} \psi \vee\left\langle\varphi_{2}\right\rangle_{K} \psi \vee\left\langle\varphi_{1}\right\rangle_{K}\left\langle\varphi_{2}\right\rangle_{K} \psi \vee\left\langle\varphi_{2}\right\rangle_{K}\left\langle\varphi_{1}\right\rangle_{K} \psi$.

Proof. For the direction from left to right, suppose that $\langle\mathfrak{M}, w\rangle=\left\langle\left\{\varphi_{1}, \varphi_{2}\right\}\right\rangle_{C} \psi$. Then there exists a world $v$ in $\mathfrak{M}$ such that $w\left(R_{\varphi_{1}} \cup R_{\varphi_{2}}\right)^{*} v$ and $\langle\mathfrak{M}, v\rangle \models \psi$. We distinguish the following two cases:

(a) $\mathfrak{M}=\varphi_{1} \leftrightarrow \varphi_{2} ;$

(b) $\langle\mathfrak{M}, u\rangle \mid=\left(\varphi_{1} \wedge \neg \varphi_{2}\right) \vee\left(\varphi_{2} \wedge \neg \varphi_{1}\right)$ for some world $u$.

For (a), it is clear that $R_{\varphi_{1}}=R_{\varphi_{2}}=R_{\varphi_{1}} \cup R_{\varphi_{2}}$ and, as each $R_{\varphi_{i}}$ is reflexive and transitive, it holds that $\left(R_{\varphi_{1}} \cup R_{\varphi_{2}}\right)^{*}=R_{\varphi_{i}}$ for every $i \in\{1,2\}$. Hence, $w\left(R_{\varphi_{1}} \cup R_{\varphi_{2}}\right)^{*} v$ implies that $w R_{\varphi_{i}} v$ which yields $\langle\mathfrak{M}, w\rangle \mid=\left\langle\varphi_{i}\right\rangle_{K} \psi$ for every $i \in\{1,2\}$. Consequently, it holds that $\langle\mathfrak{M}, w\rangle \models\left\langle\varphi_{1}\right\rangle_{K} \psi \vee\left\langle\varphi_{2}\right\rangle_{K} \psi \vee\left\langle\varphi_{1}\right\rangle_{K}$ $\left\langle\varphi_{2}\right\rangle_{K} \psi \vee\left\langle\varphi_{2}\right\rangle_{K}\left\langle\varphi_{1}\right\rangle_{K} \psi$

For $(b)$, we show that the relation $R=\left(R_{\varphi_{1}} \cup R_{\varphi_{2}}\right) \circ\left(R_{\varphi_{1}} \cup R_{\varphi_{2}}\right)$ is a universal relation. Let $t, s \in W$ be two worlds. We show that $t R s$. We will use the world $u$ as a linking point between $s$ and $t$. We know that each $R_{\varphi_{i}}$ is of the form $\llbracket \varphi_{i} \rrbracket \otimes \llbracket \neg \varphi_{i} \rrbracket$ (similar to Example 5 if we take $p=\varphi_{1}$ and $q=\varphi_{2}$ ). Hence, since $\langle\mathfrak{M}, u\rangle \models\left(\varphi_{1} \wedge \neg \varphi_{2}\right) \vee\left(\varphi_{2} \wedge \neg \varphi_{1}\right)$, it will belong to the upper cluster according to the first relation among $R_{\varphi_{1}}$ and $R_{\varphi_{2}}$, while it will belong to the lower cluster according to the second relation. Therefore $t\left(R_{\varphi_{1}} \cup R_{\varphi_{2}}\right) u$ and similarly $u\left(R_{\varphi_{1}} \cup R_{\varphi_{2}}\right) s$. Hence, it holds that $t R s$. Since $t$ and $s$ were chosen arbitrarily we can infer that $R=W \times W$, and as $R$ is reflexive and transitive we have that $R^{*}=R$. Now $w\left(R_{\varphi_{1}} \cup R_{\varphi_{2}}\right)^{*} v$ implies that $w R v$. This means that the world $v$ can be reached from $w$ in two steps, i.e., it holds that $w R_{\varphi_{1}} \circ R_{\varphi_{1}} v, w R_{\varphi_{2}} \circ R_{\varphi_{2}} v, w R_{\varphi_{2}} \circ R_{\varphi_{1}} v$ or $w R_{\varphi_{1}} \circ R_{\varphi_{2}} v$. We obtain the first two since each $R_{\varphi_{i}}$ is transitive, which means that $v$ can be reached in one step as well, whereas the second two cases do not simplify further.

For each of the above case it holds that $\langle\mathfrak{M}, w\rangle=\left\langle\varphi_{1}\right\rangle_{K} \psi \vee\left\langle\varphi_{2}\right\rangle_{K} \psi \vee\left\langle\varphi_{1}\right\rangle_{K}\left\langle\varphi_{2}\right\rangle_{K} \psi \vee\left\langle\varphi_{2}\right\rangle_{K}\left\langle\varphi_{1}\right\rangle_{K} \psi$.

For the other direction from right to left, suppose that $\langle\mathfrak{M}, w\rangle \not \models\left\langle\left\{\varphi_{1}, \varphi_{2}\right\}\right\rangle_{C} \psi$. Then, it holds that $\langle\mathfrak{M}, w\rangle \models\left[\left\{\varphi_{1}, \varphi_{2}\right\}\right]_{C} \neg \psi$. By Proposition 5 we infer that $\langle\mathfrak{M}, w\rangle \models\left[\varphi_{1}\right]_{K} \neg \psi \wedge\left[\varphi_{2}\right]_{K} \neg \psi \wedge\left[\varphi_{1}\right]_{K}\left[\varphi_{2}\right]_{K} \neg \psi \wedge$ $\left[\varphi_{2}\right]_{K}\left[\varphi_{1}\right]_{K} \neg \psi$. As a result we obtain that $\langle\mathfrak{M}, w\rangle \not \models\left\langle\varphi_{1}\right\rangle_{K} \psi \vee\left\langle\varphi_{2}\right\rangle_{K} \psi \vee\left\langle\varphi_{1}\right\rangle_{K}\left\langle\varphi_{2}\right\rangle_{K} \psi \vee\left\langle\varphi_{2}\right\rangle_{K}\left\langle\varphi_{1}\right\rangle_{K} \psi$.

Lemma 3. Let $\varphi_{1}$ and $\varphi_{2}$ be two formulas in the language of $S 5^{r}$. Then the formula $\left[\varphi_{1}, \varphi_{2}\right]_{C} p \leftrightarrow \psi(p)$ with $\psi(p)=\left[\varphi_{1}\right]_{K} p \wedge\left[\varphi_{2}\right]_{K} p \wedge\left[\varphi_{1}\right]_{K}\left[\varphi_{2}\right]_{K} p \wedge\left[\varphi_{2}\right]_{K}\left[\varphi_{1}\right]_{K} p$ is a relational modal definition for the language of $S 5^{r}$.

Proof. We need to show the existence of a first-order formula $\Psi_{\varphi_{1}, \varphi_{2}}(x, y)$ with two free variables $x$ and $y$ that uses only predicates from $\operatorname{ST}_{x}(\psi(p))$ such that for every $\chi \in \mathrm{S5}^{r}$ and every pointed model $\langle\mathfrak{M}, w\rangle$, it holds that $\langle\mathfrak{M}, w\rangle \models(\forall y)\left(\Psi_{+}(x, y) \Rightarrow \operatorname{ST}_{y}(\psi)\right)$ iff $\mathfrak{M} \models \operatorname{ST}_{x}(\psi(\chi))\left[x \mapsto c_{w}\right]$ (cf. Definition 1).

Let $\Psi_{\varphi_{1}, \varphi_{2}}(x, y)$ be such that $(v, w) \in R_{\left\{\varphi_{1}, \varphi_{2}\right\}}$ iff $\mathfrak{M} \models \Psi_{\varphi_{1}, \varphi_{2}}(x, y)\left[x \mapsto c_{v}, y \mapsto c_{w}\right]$, where

$$
R_{\left\{\varphi_{1}, \varphi_{2}\right\}}=\left(R_{\varphi_{1}} \cup R_{\varphi_{2}}\right) \circ\left(R_{\varphi_{1}} \cup R_{\varphi_{2}}\right) .
$$

Moreover, let $\mathfrak{M}$ be a model and let $w$ be a world in $\mathfrak{M}$. It suffices to show the following equivalence: $\mathfrak{M} \models(\forall y)\left(x R_{\left\{\varphi_{1}, \varphi_{2}\right\}} y \Rightarrow P(y)\right)\left[x \mapsto c_{w}\right]$ iff the following four conditions are satisfied: 
- $\mathfrak{M}=(\forall y)\left(x R_{\varphi_{1}} y \Rightarrow P(y)\right)\left[x \mapsto c_{w}\right]$;

- $\mathfrak{M} \models(\forall y)\left(x R_{\varphi_{2}} y \Rightarrow P(y)\right)\left[x \mapsto c_{w}\right]$

- $\mathfrak{M} \models(\forall y, z)\left(\left(x R_{\varphi_{1}} z \wedge z R_{\varphi_{2}} y\right) \Rightarrow P(y)\right)\left[x \mapsto c_{w}\right]$; and

- $\mathfrak{M} \models(\forall y, z)\left(\left(x R_{\varphi_{2}} z \wedge z R_{\varphi_{1}} y\right) \Rightarrow P(y)\right)\left[x \mapsto c_{w}\right]$,

where $\left[x \mapsto c_{w}\right]$ substitutes the occurrences of the free variable $x$ with the constant $c_{w}$ which is interpreted as $w$ in $\mathfrak{M}$ extended with dedicated constants, one for every world.

The equivalence is a direct application of the following equality

$$
\left(R_{\varphi_{1}} \cup R_{\varphi_{2}}\right) \circ\left(R_{\varphi_{1}} \cup R_{\varphi_{2}}\right)=R_{\varphi_{1}} \cup R_{\varphi_{2}} \cup\left(R_{\varphi_{2}} \circ R_{\varphi_{1}}\right) \cup\left(R_{\varphi_{1}} \circ R_{\varphi_{2}}\right) .
$$

It suffices to show that $R_{\varphi_{1}} \cup R_{\varphi_{2}} \subseteq\left(R_{\varphi_{2}} \circ R_{\varphi_{1}}\right) \cup\left(R_{\varphi_{1}} \circ R_{\varphi_{2}}\right)$. But this is immediate since each $R_{\varphi}$ is a reflexive relation.

The reduction of the common knowledge modality for a finite set $\Phi=\left\{\varphi_{1}, \ldots, \varphi_{n}\right\}$ of agents is recursively defined as a function $\mathrm{Rd}$ as follows, where $n \geq 3$ :

$$
\begin{aligned}
\operatorname{Rd}\left(\left[\varphi_{1}\right]_{C} p\right) & :=\left[\varphi_{1}\right]_{K} p \\
\operatorname{Rd}\left(\left[\varphi_{1}, \varphi_{2}\right]_{C} p\right):= & {\left[\varphi_{1}\right]_{K} p \wedge\left[\varphi_{2}\right]_{K} p \wedge\left[\varphi_{1}\right]_{K}\left[\varphi_{2}\right]_{K} p \wedge } \\
& {\left[\varphi_{2}\right]_{K}\left[\varphi_{1}\right]_{K} p } \\
\operatorname{Rd}\left(\left[\varphi_{1}, \ldots, \varphi_{n}\right]_{C} p\right):= & \operatorname{Rd}\left(\left[\operatorname{Rd}\left(\left[\varphi_{1}, \ldots, \varphi_{n-1}\right]_{C} p\right), \varphi_{n}\right]_{C}\right)
\end{aligned}
$$

Note that here we implicitly assume that each $\varphi_{i}$ belongs to the language of $\mathrm{S}^{r}$, i.e., $\varphi_{i}$ does not contain the modality for common knowledge. The reduction for arbitrary formulas $\Phi=\left\{\varphi_{1}, \ldots, \varphi_{n}\right\}$, where the $\varphi_{i}$-s may include the common knowledge modality, is a simple application of the reduction step by step. It can readily be seen that the function Rd yields a formula in the language of $\mathrm{S}^{r}$.

Theorem 4. Let $\Phi$ be a finite set of formulas in the language of $S 5^{r}$. Then the formula $[\Phi]_{C} p \leftrightarrow$ $\operatorname{Rd}\left([\Phi]_{C} p\right)$ is a relational modal definition for the language of $S 5^{r}$.

Proof. The case where $|\Phi|=1$ is trivial; and the case where $|\Phi|=2$ follows from Lemma 3 . Let $\Phi=$ $\left\{\varphi_{1}, \ldots, \varphi_{n}\right\}$ with $n \geq 3$. We show that $\left[\varphi_{1}, \ldots, \varphi_{n}\right]_{C} p \leftrightarrow \operatorname{Rd}\left(\left[\varphi_{1}, \ldots, \varphi_{n}\right]_{C} p\right)$ is a relational modal definition. It holds that

$$
\operatorname{Rd}\left(\left[\varphi_{1}, \ldots, \varphi_{n}\right]_{C} p\right)=\operatorname{Rd}\left(\left[\operatorname{Rd}\left(\left[\varphi_{1}, \ldots, \varphi_{n-1}\right]_{C} p\right), \varphi_{n}\right]_{C} p\right)
$$

Let $\gamma=\operatorname{Rd}\left(\left[\varphi_{1}, \ldots, \varphi_{n-1}\right]_{C} p\right)$. It can readily be seen that $\gamma$ is a formula in the language $\mathrm{S}^{r}$. Then $\operatorname{Rd}\left(\left[\varphi_{1}, \ldots, \varphi_{n}\right]_{C} p\right) \leftrightarrow \operatorname{Rd}\left(\left[\gamma, \varphi_{n}\right]_{C} p\right)$ and by Lemma 3 is equivalent to $[\gamma]_{K} p \wedge\left[\varphi_{2}\right]_{K} p \wedge[\gamma]_{K}\left[\varphi_{2}\right]_{K} p \wedge$ $\left[\varphi_{2}\right]_{K}[\gamma]_{K} p$. Again by Lemma 3 , it is a relational modal definition.

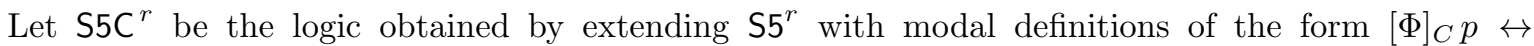
$\operatorname{Rd}\left([\Phi]_{C} p\right)$, where $\Phi$ ranges over sets of formulas in the language of $\mathrm{S}^{r}$ and the function $\mathrm{Rd}$ is defined as above. We obtain the following result.

Theorem 5. The modal logic S5C ${ }^{r}$ is sound and complete w.r.t. the class of all basic structures.

The proof of the theorem follows from theorems 1,2 and 4.

Theorem 6. The logic $55 C^{r}$ is decidable.

Proof. Proof follows from decidability of the logic $S 5^{r}$ and reducibility of an arbitrary $\mathrm{S5D}^{r}$ formula to a $\mathrm{S}^{r}$ formula. 


\subsection{Topological Semantics}

Here we introduce the topological semantics for the logic $\mathrm{S}_{5} \mathrm{C}^{r}$. Following the ideas from [30] we interpret the common knowledge modality on the intersection topology. More formally the satisfaction of the formula $\left[\varphi_{1}, \ldots, \varphi_{n}\right]_{C} \psi$ in the topological model $\mathfrak{T}=(X, \tau, V)$ at a point $x$ is defined by the following clause:

- $\mathfrak{T}, x \models\left[\varphi_{1}, \ldots, \varphi_{n}\right]_{C} \psi$ iff $x \in \operatorname{Int}_{\cap} \llbracket \psi \rrbracket$, where $\operatorname{Int}_{\cap}$ is the interior operator of the intersection topology $\left(X, \tau_{\cap}\right)$ with $\tau_{\cap}=\bigcap_{i=1 . . n} \tau_{\varphi_{i}}$.

Theorem 7. The modal logic $S 5 C^{r}$ is sound and complete w.r.t. the class of all topological structures.

Proof. We skip soundness since it follows by straightforward check. For completeness assume that $\alpha \notin$ $\mathrm{S}_{5} \mathrm{C}^{r}$. By Theorem 5 there is a basic structure $\mathfrak{M}=(W, V)$ and a point $w$ such that $w \not \forall \alpha$. Let us consider all generated relations $R_{\varphi}$ which we need to evaluate the formula $\alpha$ in the model $\mathfrak{M}$. Formally we take a set $\Gamma$ of all formulas $\varphi$ such that there exists a subformula of $\alpha$ of the form $[\varphi]_{K} \psi$ or $\left[\varphi_{1}, \ldots, \varphi_{n}\right]_{C} \psi$ with $\varphi=\varphi_{k}$ for some $k$. Let us consider the model $\left(W,\left\{R_{\varphi}\right\}_{\varphi \in \Gamma}, V\right)$. It follows from the semantics of the logic S5C ${ }^{r}$ that $\mathfrak{M}, w \models \alpha$ iff $\left(W,\left\{R_{\varphi}\right\}, V\right), w \models \alpha$ when each $[\varphi]_{K}$ is evaluated on the corresponding relation $R_{\varphi}$ as a standard box operator and $\left[\varphi_{1}, \ldots, \varphi_{n}\right]_{C} \psi$ is evaluated as a standard box operator on the reflexive and transitive closure $\left(\bigcup_{i=1,2, \ldots n} R_{\varphi_{i}}\right)^{*}$. Now by Alexandroff connection 2 we have the following iff condition: $\left(W,\left\{R_{\varphi}\right\}_{\varphi \in \Gamma}, V\right), w \models \alpha$ iff $\left(W,\left\{\tau_{R_{\varphi}}\right\}_{\varphi \in \Gamma}, V\right), w \models \alpha$ with common knowledge modality being evaluated on $\tau_{R_{\left(\cup_{i=1,2, . . n} R_{\varphi_{i}}\right)^{*}}}$. Again by the property of Alexandroff connection 1 we know that $\tau_{\left(\bigcup_{i=1,2, \ldots, n} R_{\varphi_{i}}\right)^{*}}=\bigcap_{i=1,2, . ., n} \tau_{R_{\varphi_{i}}}$. By lemma 1 we conclude that $\tau_{\left(\bigcup_{i=1,2, \ldots, n} R_{\varphi_{i}}\right)^{*}}=\bigcap_{i=1,2, . ., n} \tau_{\varphi_{i}}$ which finishes the proof.

\section{The Modal Logic S5D ${ }^{r}$}

In this section, we introduce the modal logic $\mathrm{S}^{2} \mathrm{D}^{r}$ which is an extension of $\mathrm{S}^{r}$ with modalities for distributed hypotheses that are analogous to modalities for distributed knowledge. Distributed knowledge in modal logic is a well-known notion; standard references include [11, 18, and for a more recent discussion, see [12, 20]. We leave the prove of the completeness results for this logic for future work. We show how distributed hypotheses can be used to represent the knowledge of an agent whose epistemic capacity corresponds to any system containing S4.

Let $\Pi$ be a countably infinite set of atomic propositions. Formulas $\varphi$ of $\mathrm{S}^{2} \mathrm{D}^{r}$ are defined inductively over $\Pi$ by the following grammar:

$$
\varphi::=p|\neg \varphi| \varphi \vee \varphi\left|[\varphi]_{K} \varphi\right|[\Phi]_{D} \varphi
$$

where $p$ ranges over atomic propositions in $\Pi$, and $\Phi$ over finite sets of $\mathrm{S}^{2} \mathrm{D}^{r}$-formulas.

Formulas of $\mathrm{S}^{r} \mathrm{D}^{r}$ are evaluated in basic structures as well. The operators $[\Phi]_{D}$ are necessities depending on the formulas in $\Phi$. The semantics of $[\Phi]_{D}$ is based on the relations $R_{\varphi}$, where $\varphi \in \Phi$, as follows. Let $\mathfrak{M}=(W, V)$ be a basic structure. The logical consequence relation ' $=$ ' and the relations $R$ for formulas of $\mathrm{S}^{2} \mathrm{D}^{r}$ are defined as for $\mathrm{S}^{r}$ but extended with the following clauses: For all $\mathrm{S}^{2} \mathrm{D}^{r}$-formulas and all finite sets $\Phi$ of $\mathrm{S}^{2} \mathrm{D}^{r}$-formulas,

• $\mathfrak{M}, w \mid=[\Phi]_{D} \psi$ iff for all $v \in W$ with $(w, v) \in R_{\Phi}, \mathfrak{M}, v \models \psi$,

where $R_{\Phi}=\bigcap_{\varphi \in \Phi} R_{\varphi}$.

The following proposition shows how any finite preorder can be represented as an intersection of one-step frames. This hints at the possibility that an arbitrary modal operator can be represented as a distributed knowledge of some hypothetical knowledge operator. Here we assume that knowledge operators are box-modalities for logics in the interval $[S 4, S 5]$. Below we show a version of this claim.

Proposition 7. Let $W=\left\{w_{1}, \ldots, w_{k}\right\}$ be a set. Let $R$ be a preorder over $W$. For all $i \in\{1, \ldots, k\}$, let $R_{i}=\left(W \backslash R\left(w_{i}\right)\right) \otimes R\left(w_{i}\right)$. Then it holds that $R=\bigcap_{i=1 . . k} R_{i}$. 
Intuitively, the proposition can be understood as follows. Observe that a preorder $R$ induces a partial order (i.e. an antisymmetic preorder) on the set of $R$-clusters, which are sets of points fully connected by $R$. In other words, $R$ gives rise to a collection of directed graphs whose nodes are $R$-clusters. Note that the graph is loopless (and thus antisymmetric). Now, if $R$ is total, all points are connected which gives rise to just one such graph. If, additionally, $R$ is 'one-step', the graph consists of merely two nodes. Intersecting one-step total preorders has the effect of erasing directed edges from the universal relation. Note that the intersection of preorders is again a preorder. Proposition 7 shows that by intersecting a certain selection of one-step total preorders, we can "carve out" the desired preorder. The following example illustrates the scenario.

Example 6. Let $W=\{x, y, z\}$ be a set and $R=\{(x, y),(x, z)\} \cup i d(W)$. It is readily checked that $R$ is a reflexive and transitive relation. Now let $R_{w}=(W \backslash R(w)) \otimes R(w)$ for all $w \in W$. That is, according to Equation (1), we have $R_{x}=W \times W, R_{y}=\{(x, y),(z, y),(x, z),(z, x)\} \cup i d(W)$ and $R_{z}=$ $\{(y, z),(x, z),(x, y),(y, x)\} \cup i d(W)$. Intersecting these relations we obtain $R_{x} \cap R_{y} \cap R_{z}=\{(x, y),(x, z)\} \cup$ id $(W)$, which is equivalent to $R$.

The intersection in Proposition 7 reminds us on the relations $R_{\Phi}$ determined by a finite set $\Psi$ of $\mathrm{S}_{5} \mathrm{D}^{r}$-formulas in a model. In fact, this is the connection we seek to establish in order to represent the knowledge of an agent as distributed knowledge. In the following, we state how this is done.

Take an arbitrary uni-modal logic L between S4 and S5 (whose satisfaction relation is denoted by $\models_{\mathrm{L}}$ ). The necessity operator ' $\square$ ' of $\mathrm{L}$ is thought of as representing the knowledge of the agent. Note that the system L contains the axioms ( $\mathrm{T}$ ) and (4), each of which represent important epistemic properties, namely, veridicality and positive introspection, respectively. Of course, L may contain other axioms, in fact, any axiom that can be derived in system $\mathrm{S} 5$. We assume that $\mathrm{L}$ is determined by a class $\mathcal{C}$ of Kripke structures (i.e., the theorems of $\mathrm{L}$ are exactly the formulas that are valid on all structures in $\mathcal{C}$ ). Clearly, the structures in $\mathcal{C}$ are reflexive and transitive. What we require as a precondition is that $\mathrm{L}$ has the finite-model property w.r.t. $\mathcal{C}$. This means that, if a formula $\varphi$ is not a theorem of $\mathrm{L}$ then there is a finite Kripke structure $\mathfrak{M}^{k}$ in $\mathcal{C}$ that falsifies $\varphi$, i.e. $\mathfrak{M}^{k}, w \not \models \varphi$ for some world $w$ in $\mathfrak{M}^{k}$.

Before we can state the theorem, we need one more auxiliary notion. Let $\mathfrak{M}^{k}=(W, R, V)$ be a finite Kripke structure such that the relation $R$ is a preorder. We say that the valuation function $V$ covers $R$ if for every world $w \in W$, there is an atomic proposition $p_{w}$ such that $V\left(p_{w}\right)=R(w)$, i.e. the $R$-image at $w$.

Theorem 8. Let $\mathcal{C}$ be a class of Kripke structures whose relations are preorders. Let $\mathfrak{M}^{k}=(W, R, V)$ be a finite structure from $\mathcal{C}$ such that $V$ covers $R$. Let $\mathfrak{M}=(W, V)$ be a basic structure and let $w \in W$ be a world. Let $\varphi$ be a Boolean formula over $\Pi$. Then, there is a finite set $\Psi$ of atomic propositions such that the following are equivalent:

(i) $\mathfrak{M}^{k}, w \mid{ }_{L} \square \varphi$;

(ii) $\mathfrak{M}, w \models S 5 D^{r}[\Psi]_{D} \varphi$.

Proof. For every $w \in W$, select an atomic proposition $p_{w}$ such that $V\left(p_{w}\right)=R(w)$. Note that such $p_{w}$ exists since $V$ covers $R$. Set $\Psi=\left\{p_{w} \mid w \in W\right\}$. Using Lemma 7 the equivalence of (i) and (ii) can be shown by induction on the structure of $\varphi$.

We remark that the theorem can be generalized since the condition of using finite models is a bit too strict. Recall the metaphor that views a preorder $R$ as a collection of loopless graphs whose nodes are $R$-clusters. What is actually required is that the collection of graphs and the graphs themselves are finite. So, we can still find a finite intersection of relations as desired.

The following example illustrates Theorem 8 and discusses the presented notions.

Example 7. Consider the Kripke model $\mathfrak{M}^{k}=(W, R, V)$, where $W$ and $R$ are as in Example 6 , and $V(p)=\{x, z\}$ and $V(q)=\{z\}$. Clearly, $\mathfrak{M}^{k}$ is not an S5-model as $R$ is not symmetric. Let $\varphi .2, \varphi .3, \varphi .4$ be the instances of the axioms (.2), (.3) and (.4) as shown above. It turns out that only $\varphi .3$ holds at $x$, but not $\varphi .2$ nor $\varphi .4$. In fact, $\varphi$.3 holds at all worlds in $\mathfrak{M}^{k}$. Let us assume that the box (i.e., the epistemic capacity of the agent) is characterized by the system $S_{4.3 .}$

Now label the worlds with fresh atomic propositions $p_{x}, p_{y}, p_{z}$, i.e., we set $V^{\prime}\left(p_{w}\right)=\{w\}$ for all $w \in W$. Notice that $V^{\prime}$ covers $R$. Let $R_{p_{x}}, R_{p_{y}}, R_{p_{z}}$ be the relations determined by the basic structure 
$\mathfrak{M}=\left(W, V^{\prime}\right)$ and the fresh propositions. Notice that $R_{p_{w}}$ equals $R_{w}$ from Example 6 , for every $w \in W$. Thus $R_{p_{x}} \cap R_{p_{y}} \cap R_{p_{z}}=R$. Now it is immediate that $\mathfrak{M}, w=\left[\left\{p_{x}, p_{y}, p_{z}\right\}\right]_{D} \varphi$ iff $\mathfrak{M}^{k}, w \models \square \varphi$, for all $w \in W$ and all propositional formulas $\varphi$ without occurrence of any of $p_{x}, p_{y}$ and $p_{z}$. In other words, $\left[\left\{p_{x}, p_{y}, p_{z}\right\}\right]_{D}$ simulates the S4.3-box. We can see $p_{x}, p_{y}, p_{z}$ as hypotheses that another agent has to adopt in order to know what the S4.3-agent knows.

In some cases, we have an alternative to introducing fresh propositions even though $V$ does not cover $R$. This means that $V$ covering $R$ is a sufficient but not necessary condition for Theorem 8 . Here $\neg p$ and $q$ are hypotheses so that $[\{\neg p, q\}]_{D}$ simulates S4.3-box as well. That is, hypotheses do not need to be atomic propositions. Moreover, (parts of) hypotheses may occur in the conclusion as in $(W, V), x \models[\{\neg p, q\}]_{D} \varphi .3$.

The reduction of the distributive knowledge modality for a finite set $\Phi=\left\{\varphi_{1}, \ldots, \varphi_{n}\right\}$ of agents is recursively defined as a function $\mathrm{Rd}$ as follows, where $n \geq 3$ :

$$
\begin{aligned}
\operatorname{Rd}\left(\left[\varphi_{1}\right]_{D} p\right) & :=\left[\varphi_{1}\right]_{K} p \\
\operatorname{Rd}\left(\left[\varphi_{1}, \varphi_{2}\right]_{D} p\right) & :=\left[\varphi_{2}\right]_{K} p \vee\left(\varphi_{1} \wedge\left[\varphi_{2}\right]_{K}\left(\varphi_{1} \rightarrow p\right)\right. \\
\operatorname{Rd}\left(\left[\varphi_{1}, \ldots, \varphi_{n}\right]_{D} p\right) & :=\operatorname{Rd}\left(\left[\operatorname{Rd}\left(\left[\varphi_{1}, \ldots, \varphi_{n-1}\right]_{D} p\right), \varphi_{n}\right]_{D} p\right)
\end{aligned}
$$

Here we assume that each $\varphi_{i}$ belongs to the language of $\mathrm{S} 5^{r}$, i.e., $\varphi_{i}$ does not contain the distributive knowledge modality although the reduction for arbitrary formulas $\Phi=\left\{\varphi_{1}, \ldots, \varphi_{n}\right\}$, where the $\varphi_{i}$-s may include the distributive knowledge modality, is a simple application of the reduction step by step. It can readily be seen that the function $\mathrm{Rd}$ yields a formula in the language of $\mathrm{S}^{r}$.

Theorem 9. Let $\Phi$ be a finite set of formulas in the language of $S 5^{r}$. Then the formula $[\Phi]_{D} p \leftrightarrow$ $\operatorname{Rd}\left([\Phi]_{D} p\right)$ is a relational modal definition for the language of $\mathrm{S5}^{r}$.

Let $\mathrm{S} 5 \mathrm{D}^{r}$ be the logic obtained by extending $\mathrm{S}^{r}$ with modal definitions of the form $[\Phi]_{D} p \leftrightarrow$ $\operatorname{Rd}\left([\Phi]_{D} p\right)$, where $\Phi$ ranges over sets of formulas in the language of $\mathrm{S}^{r}$ and the function $\mathrm{Rd}$ is defined as above. We obtain the following result.

Theorem 10. The modal logic S5D ${ }^{r}$ is sound and complete w.r.t. the class of all basic structures.

The proof of the theorem follows from the fact that the modal logic $\mathrm{S} 5^{r}$ is complete and theorems 1 and 9. We also imply decidability of the logic since all formulas are reduced to $S 5^{r}$ formulas.

Theorem 11. The logic $S 5 D^{r}$ is decidable.

Proof. Proof follows from decidability of the logic $S 5^{r}$ and reducibility of an arbitrary S5D $^{r}$ formula to a $S 5^{r}$ formula.

\subsection{Topological Semantics}

Topological semantics for the logic $\mathrm{S}^{2} \mathrm{D}^{r}$ is provided by the topological sum in other words we interpret the distributed knowledge modality by the interior operator of the topological sum of the corresponding topologies. More formally the satisfaction of the formula $\left[\varphi_{1}, \ldots, \varphi_{n}\right]_{D} \psi$ in the topological model $\mathfrak{T}=$ $(X, \tau, V)$ at a point $x$ is defined by the following clause:

- $\mathfrak{T}, x=\left[\varphi_{1}, \ldots, \varphi_{n}\right]_{D} \psi$ iff $x \in \operatorname{Int}_{\sqcup} \llbracket \psi \rrbracket$, where $\operatorname{Int}_{\sqcup}$ is the interior operator of the topological sum of topologies $\left(X, \tau_{\sqcup}\right)$ with $\tau_{\sqcup}=\bigsqcup_{i=1 . . n} \tau_{\varphi_{i}}$.

Theorem 12. The modal logic $S 5 D^{r}$ is sound and complete w.r.t. the class of all topological structures.

Proof. Again we skip soundness since it follows by easy check. For completeness assume that $\alpha \notin \mathrm{S} 5 \mathrm{D}^{r}$. By Theorem 10 there is a basic structure $\mathfrak{M}=(W, V)$ and a point $w$ such that $w \forall \neq \alpha$. Let us consider all generated relations $R_{\varphi}$ which we need to evaluate the formula $\alpha$ in the model $\mathfrak{M}$. Formally we take a set $\Gamma$ of all formulas $\varphi$ such that there exists a subformula of $\alpha$ of the form $[\varphi]_{K} \psi$ or $\left[\varphi_{1}, \ldots, \varphi_{n}\right]_{D} \psi$ with $\varphi=\varphi_{k}$ for some $k$. Let us consider the model $\left(W,\left\{R_{\varphi}\right\} \varphi \Gamma, V\right)$. It follows from the semantics of the logic $\operatorname{S5D}^{r}$ that $\mathfrak{M}, w \models \alpha$ iff $\left(W,\left\{R_{\varphi}\right\}, V\right), w \models \alpha$ when each $[\varphi]_{K}$ is evaluated on the corresponding relation $R_{\varphi}$ as a standard box operator and $\left[\varphi_{1}, \ldots, \varphi_{n}\right]_{D} \psi$ is evaluated as a standard box operator on $\bigcap_{i=1,2, \ldots n} R_{\varphi_{i}}$. Now by Alexandroff connection 2 we have the following iff 
condition: $\left(W,\left\{R_{\varphi}\right\}_{\varphi \in \Gamma}, V\right), w \models \alpha$ iff $\left(W,\left\{\tau_{R_{\varphi}}\right\}_{\varphi \in \Gamma}, V\right), w \models \alpha$ with distributed knowledge modality being evaluated on $\tau_{R_{n_{i=1,2}} R_{\varphi_{i}}}$. Again by the property of Alexandroff connection 11 we know that $\tau_{\bigcap_{i=1,2, . ., n} R_{\varphi_{i}}}=\bigsqcup_{i=1,2, . ., n} \tau_{R_{\varphi_{i}}}$. By lemma 1 we conclude that $\tau_{\bigcap_{i=1,2, . ., n} R_{\varphi_{i}}}=\bigsqcup_{i=1,2, . ., n} \tau_{\varphi_{i}}$ which finishes the proof.

\section{Conclusion}

In this paper, we continue investigating the logic $\mathrm{S}^{r}$, which is both an extension of $\mathrm{S} 5$ and a special case of Chella's Conditional Logic. We recall a Kripke completeness result and prove the topological completeness for the logic $\mathrm{S}^{r}$. In the second part of the paper, we extend the logic with modalities for common knowledge and prove Kripke and topological completeness theorems. Also we show that $\mathrm{S}_{5} \mathrm{C}^{r}$ is decidable. In the third part of the paper, we extend the logic with modalities for distributed hypotheses that are analog to modalities for distributed knowledge. Distributed hypotheses can be employed to represent the knowledge of agents whose epistemic capacity corresponds to any system containing S4. We also prove Kripke and topological completeness and decidability results for the logic S5D ${ }^{r}$. Possible directions for future work are to investigate complexity issues of the logics S5C ${ }^{r}$ and $\mathrm{S}^{2} \mathrm{D}^{r}$.

Acknowledgements: The first author was partially supported by Shota Rustaveli National Science Foundation of Georgia (SRNSFG) grant number YS17-71.

\section{References}

[1] Alexandru Baltag, Lawrence S. Moss, and Slawomir Solecki. The logic of public announcements, common knowledge, and private suspicions. In Proceedings of TARK'98: the "th conference on Theoretical aspects of rationality and knowledge, TARK '98, pages 43-56, San Francisco, CA, USA, 1998. Morgan Kaufmann Publishers Inc.

[2] Jon Barwise. Three views of common knowledge. In Proceedings of the 2nd conference on Theoretical aspects of reasoning about knowledge, TARK '88, pages 365-379, San Francisco, CA, USA, 1988. Morgan Kaufmann Publishers Inc.

[3] Esakia L. Bezhanishvili, G. and Gabelaia D. Some results on modal axiomatization and definability for topological spaces. Studia Logica, 81:325-355, 2005.

[4] N. Bezhanishvili and W. van der Hoek. Structures for Epistemic Logic (Survey). Logic Group Preprint Series, 2013.

[5] P. Blackburn, M. de Rijke, and Y. Venema. Modal Logic. Cambridge University Press, Cambridge, UK, 2001.

[6] George S. Boolos. The Logic of Provability. Cambridge University Press, 1993.

[7] Brian F. Chellas. Basic conditional logic. Journal of Philosophical Logic, 4:133-153, 1975.

[8] Brian F. Chellas. Modal Logic: An Introduction. Cambridge University Press, 1980.

[9] Leo Esakia. Weak transitivity - a restitution. In Logical Investigations, volume 8, pages 244-255, Moscow, Nauka, 2001. In Russian.

[10] Leo Esakia. Around provability logic. Annals of Pure and Applied Logic, 161(2):174-184, 2009. Festschrift on the occasion of Franco Montagna's 60th birthday.

[11] Ronald Fagin, Joseph Y. Halpern, Yoram Moses, and Moshe Y. Vardi. Reasoning about Knowledge. The MIT Press, 1995.

[12] Jelle Gerbrandy. Distributed knowledge. In Twendial'98: Formal Semantics and Pragmatics of Dialogue, TWLT 13, pages 111-124. Universiteit Twente, Enschede, 1998. 
[13] R. Goldblatt and S. Thomason. Axiomatic classes in propositional modal logic. In John Crossley, editor, Algebra and Logic, volume 450 of Lecture Notes in Mathematics, pages 163-173. Springer Berlin / Heidelberg, 1975.

[14] A.; Herzig, A.; deLima and T. E. Lorini. On the dynamics of institutional agreements. In Proceedings of Knowledge, Rationality and Action 2009, pages 923-957, 2009.

[15] David Lewis. Convention: A Philosophical Study. Harvard University Press, Cambridge, Massachusetts, 1969.

[16] David Lewis. Counterfactuals. Harvard University Press, Cambridge, Massachusetts, 1973. revised printing Blackwell.

[17] J. C. C. McKinsey and A. Tarski. The algebra of topology. Annals of Mathematics, 45:141-191, 1944.

[18] J. Ch. Meyer and W. van der Hoek. Epistemic Logic for AI and Computer Science, volume 41 of Cambridge Tracts in Theoretical Computer Science. Cambridge University Press, Cambridge, England, 1995.

[19] Jan A. Plaza. Logics of public communications. In Proceedings of the 4th International Symposium on Methodologies for Intelligent Systems, pages 201-216. Oak Ridge National Laboratory, ORNL/DSRD-24, 1989.

[20] Floris Roelofsen. Distributed knowledge. Journal of Applied Non-Classical Logics, 17(2):255-273, 2007.

[21] H. Sahlqvist. Completeness and correspondence in the first and second order semantics for modal logics. In Proceedings of the 3rd Scandinavian Logic Symposium, 1973, pages 110-143. North-Holland Publishing Company, 1975.

[22] Robert Stalnaker. A theory of conditionals. Studies in Logical Theory, pages 98-112, 1968.

[23] Levan Uridia and Dirk Walther. An epistemic logic with hypotheses. In Hans van Ditmarsch, Jérôme Lang, and Shier Ju, editors, Logic, Rationality, and Interaction, volume 6953 of Lecture Notes in Computer Science, pages 286-299. Springer Berlin / Heidelberg, 2011.

[24] Levan Uridia and Dirk Walther. Completeness by modal definitions. In Proceedings of IBERAMIA18: the 16th Ibero-American Conference on AI 2018, volume 11238 of Lecture Notes in Artificial Intelligence, pages 67-79. Springer International Publishing, 2018.

[25] Levan Uridia and Dirk Walther. Common knowledge in epistemic logic with hypotheses. In Diego Calvanese and Luca Iocchi, editors, GCAI 2019. Proceedings of the 5th Global Conference on Artificial Intelligence, Bozen/Bolzano, Italy, 17-19 September 2019, volume 65 of EPiC Series in Computing, pages 139-151. EasyChair, 2019.

[26] Levan Uridia and Dirk Walther. Distributed knowledge in epistemic logic with hypotheses. In Proceedings of the 6th Global Conference on Artificial Intelligence 2020, Accepted for publication.

[27] Johan van Benthem. Modal frame classes revisited. Fundamenta Informaticae, 18:307-317, 1993.

[28] Johan van Benthem and Fenrong Liu. Dynamic logic of preference upgrade. Journal of Applied Non-Classical Logics, 17(2):157-182, 2007.

[29] Johann van Benthem. Rational dynamics and epistemic logic in games. International Game Theory Review, 9:13-45, 2007. Erratum reprint, Volume 9:2, 377-409.

[30] van Benthem J. and Sarenac D. The geometry of knowledge. Aspects of universal logic, 17:1-31, 2004 .

[31] Hans van Ditmarsch, Wiebe van der Hoek, and Barteld Kooi. Dynamic Epistemic Logic, volume 337 of Synthese Library: Studies in Epistemology, Logic, Methodology, and Philosophy of Science. Springer, Dordrecht, The Netherlands, 2007. 